\title{
AGN Triggering in the Infall Regions of Distant X-Ray Luminous Galaxy Clusters at $0.9<\mathrm{z} \lesssim 1.6$
}

\author{
R. Fassbender, ${ }^{1}$ R. Šuhada, ${ }^{2}$ and A. Nastasi ${ }^{1}$ \\ ${ }^{1}$ Max-Planck-Institut für Extraterrestrische Physik (MPE), Postfach 1312, Giessenbachstraße, 85741 Garching, Germany \\ ${ }^{2}$ Department of Physics, Ludwigs-Maximilians University Munich, Scheinerstraße 1, 81679 Munich, Germany \\ Correspondence should be addressed to R. Fassbender, rfassben@mpe.mpg.de
}

Received 14 November 2011; Accepted 20 March 2012

Academic Editor: Angela Bongiorno

Copyright ( $) 2012$ R. Fassbender et al. This is an open access article distributed under the Creative Commons Attribution License, which permits unrestricted use, distribution, and reproduction in any medium, provided the original work is properly cited.

\begin{abstract}
Observational constraints on the average radial distribution profile of AGN in distant galaxy clusters can provide important clues on the triggering mechanisms of AGN activity in dense environments and are essential for a completeness evaluation of cluster selection techniques in the X-ray and mm wavebands. The aim of this work is a statistical study with XMM-Newton of the presence and distribution of X-ray AGN in the large-scale structure environments of $22 \mathrm{X}$-ray luminous galaxy clusters in the redshift range $0.9<z \lesssim 1.6$ compiled by the XMM-Newton Distant Cluster Project (XDCP). To this end, the X-ray point source lists from detections in the soft band $(0.35-2.4 \mathrm{keV})$ and full band $(0.3-7.5 \mathrm{keV})$ were stacked in cluster-centric coordinates and compared to average background number counts extracted from three independent control fields in the same observations. A significant full-band (soft-band) excess of $\sim 78$ (67) X-ray point sources is found in the cluster fields within an angular distance of $8^{\prime}$ (4 Mpc) at a statistical confidence level of $4.0 \sigma(4.2 \sigma)$, corresponding to an average number of detected excess AGN per cluster environment of $3.5 \pm 0.9(3.0 \pm 0.7)$. The data point towards a rising radial profile in the cluster region $(r<1 \mathrm{Mpc})$ of predominantly lowluminosity AGN with an average detected excess of about one point source per system, with a tentative preferred occurrence along the main cluster elongation axis. A second statistically significant overdensity of brighter soft-band-detected AGN is found at cluster-centric distances of $4^{\prime}-6^{\prime}(2-3 \mathrm{Mpc})$, corresponding to about three times the average cluster radius $R_{200}$ of the systems. If confirmed, these results would support the idea of two different physical triggering mechanisms of X-ray AGN activity in dependence of the radially changing large-scale structure environment of the distant clusters. For high- $z$ cluster studies at lower spatial resolution with the upcoming eROSITA all-sky X-ray survey, the results suggest that cluster-associated X-ray AGN may impose a bias in the spectral analysis of high- $z$ systems, while their detection and flux measurements in the soft band may not be significantly affected.
\end{abstract}

\section{Introduction}

Observational studies of the connection of Active Galactic Nuclei (AGN) with the large-scale environment of massive clusters of galaxies and their mutual cosmic evolution can provide important insights into the physical conditions necessary to trigger or suppress AGN activity in galaxies. In this respect, AGN activity can be charted in dependence of the changing environments of galaxy clusters as a function of cluster-centric distance: from the dense cores, to the cluster outskirts, and further out to the matter infall regions and the surrounding cosmic web. Furthermore, the evolution of the occurrence of X-ray and radio AGN in cluster environments as a function of redshift is of key importance for the characterization and completeness evaluation of ongoing and future high- $z$ cluster surveys in the X-ray band and via the Sunyaev-Zeldovich effect (SZE) at mm wavelengths.

Numerous Chandra sample studies on the X-ray AGN content of galaxy clusters up to redshifts of about unity, for example, (e.g., [1-6]), have firmly established that the AGN fraction in clusters is significantly rising as a function of redshift. On the other hand, the cluster environment appears to suppress the occurrence of X-ray AGN activity in massive galaxies compared to a field galaxy sample at all probed redshifts so far, for example, (e.g., $[3,5,7]$ ), and the distribution of X-ray AGN is significantly less concentrated 
in terms of cluster-centric distance in comparison to radio AGN, (e.g., $[1,8,9])$. However, owing to the small number of very distant test clusters at $z>0.9$ available to these studies, the data basis at redshifts beyond unity is still very sparse, and at $z>1.3 \mathrm{X}$-ray cluster studies are limited to a single system $[10,11]$. So far, the only cluster AGN study with a sizable sample of systems at the epoch $1<z \lesssim 1.5$ was presented in [12] based on infrared selected clusters from the IRAC Shallow Cluster Survey [13], which found a continuing trend of increasing AGN fractions with redshifts in systems with an average halo mass of $\sim 10^{14} \mathrm{M}_{\odot}$.

The aim of this paper is to extend the accessible redshift regime for a sample of X-ray selected clusters to $0.9<$ $z \lesssim 1.6$ in order to perform a statistical study of the $\mathrm{X}$ ray point source excess and its radial dependence in distant $\mathrm{X}$-ray luminous systems. In contrast to the previous studies based on targeted follow-up observations with Chandra, this work is built upon archival XMM-Newton observations, in which the clusters have been serendipitously detected. This distant cluster sample and the performed X-ray point source stacking analysis are introduced in Section 2 . The results are presented in Section 3, followed by the discussion in Section 4, and conclusions in Section 5. A standard $\Lambda$ CDM cosmological model with parameters $\left(H_{0}, \Omega_{\mathrm{m}}, \Omega_{\mathrm{DE}}, w\right)=$ $\left(70 \mathrm{~km} \mathrm{~s}^{-1} \mathrm{Mpc}^{-1}, 0.3,0.7,-1\right)$ is assumed throughout this paper, which yields a median projected angular scale of $8.2 \mathrm{kpc} /{ }^{\prime \prime}$ (corresponding to $2.0^{\prime} / \mathrm{Mpc}$ ) with $<5 \%$ variation in the probed redshift interval $z=0.9-1.6$.

\section{Distant Cluster Sample and X-Ray Stacking Analysis}

2.1. Cluster Sample. This work uses the largest published sample of distant X-ray luminous galaxy clusters at redshifts $z>0.9$ to date as presented in Fassbender et al. [14]. The sample comprises $22 \mathrm{X}$-ray selected clusters in the redshift range $0.9<z \lesssim 1.6$ with a median system mass of $M_{200} \simeq$ $2 \times 10^{14} \mathrm{M}_{\odot}$. All clusters are part of the XMM-Newton Distant Cluster Project (XDCP, e.g., [14-16]), a serendipitous Xray survey focussing on the detection and study of galaxy clusters in the first half of cosmic time. Table 1 provides an overview of the considered cluster sample, including the XMM-Newton observations used for this study, their effective clean exposure time (ECT) after flare removal, and original relevant source publications for the individual clusters. (We define the ECT as the period during which all three instruments in imaging operation would collect the equivalent number of soft science photons for the particular observation.)

All clusters in this sample have by construction medium to deep XMM-Newton observations available, whereas the Chandra archive coverage of this newly constructed sample is currently less than $30 \%$. The complete coverage and high sensitivity is the main advantage to perform a first $\mathrm{X}$ ray point source study around distant X-ray clusters with XMM-Newton. A second advantage is the larger $30^{\prime}$-diameter field of view (FoV), which allows direct measurements of background counts in the same observation. XMM-Newton's spatial resolution of $5-15^{\prime \prime}$ (FWHM), on the other hand, is significantly lower than for Chandra, which implies that the central cluster core regions of up to $\sim 15^{\prime \prime} \simeq 120 \mathrm{kpc}$ cannot be properly probed for point sources in addition to the underlying detected extended X-ray emission of the cluster.

In any case, the statistical detection of a point source excess around distant clusters at $z>0.9$ is a challenging task since the average background density of X-ray point sources in the considered XMM-Newton fields is more than 20 within a $6^{\prime}$-radius $(\sim 3 \mathrm{Mpc})$ compared to an expected cluster excess of a few sources $(\sim 1.5$ for $z<0.9$ systems [4]). On the other hand, any measurable point source excess associated with $z>0.9$ clusters can be directly attributed to AGN activity with X-ray luminosities of $L_{X}>10^{43} \mathrm{erg} \mathrm{s}^{-1}$ owing to the average soft-band point source detection limit in the XMMNewton fields of $\gtrsim 10^{-15} \mathrm{erg} \mathrm{s}^{-1} \mathrm{~cm}^{-2}$.

2.2. X-Ray Data. The XMM-Newton observations used for this work are in general the original discovery fields of the clusters as listed in Table 1 (column 6), with the exception of C08 for which the deeper follow-up observation was used. The clean effective exposure time of these fields after flare removal (column 7) ranges from 8.5 to $82 \mathrm{ksec}$ with a median integration time of $19 \mathrm{ksec}$. Three of the fields contain 2-3 confirmed distant clusters inside their FoV, which are treated here as independent observations for each cluster source.

Most of the identified high- $z$ clusters are located at offaxis angles $\Theta_{\text {off }}$ between 5 and 12 arcmin from the optical axis, which was defined as the aimpoint of the PN (the most sensitive instrument) labelled as center in Figure 1. The $30^{\prime}$ diameter FoV of XMM-Newton allows to define three quasi independent background control regions inside the same XMM-Newton observation at the same off-axis angle with centers rotated by 90,180 , and 270 degrees about the optical axis as is shown in Figure 1. These fixed rotation angles to the control field centers ensure maximally spaced distances at a given off-axis angle between control and clusters fields in order to maximize the non overlapping regions between them. When comparing the cluster environment and background field point source counts, this approach (to first order) automatically accounts for (i) changes in the effective exposure time across the FoV due to vignetting, (ii) the changing PSF as a function of off-axis angle, and (iii) possible incomplete coverage beyond some cluster-centric distance in the outer radial direction due to the edge of the instrumental FoV. For the observations in which the cluster location is at small off-axis angles of $\Theta_{\text {off }}<8^{\prime}$ the control field locations were shifted back to $\Theta_{\text {off }}=8^{\prime}$ at their respective rotation angles of 90, 180, and 270 degrees. This ensures that cluster and background fields are not overlapping within their minimum separations of $>8^{\prime}$, while only slightly changing the average effective sensitivity in the control fields relative to the cluster environment.

The X-ray source detection was performed as part of the XDCP distant cluster survey as detailed in Fassbender et al [14]. Here the focus of the analysis lies on the detected point sources in the individual cluster fields, which are displayed by green circles in Figures 1 and 2. The X-ray source lists 


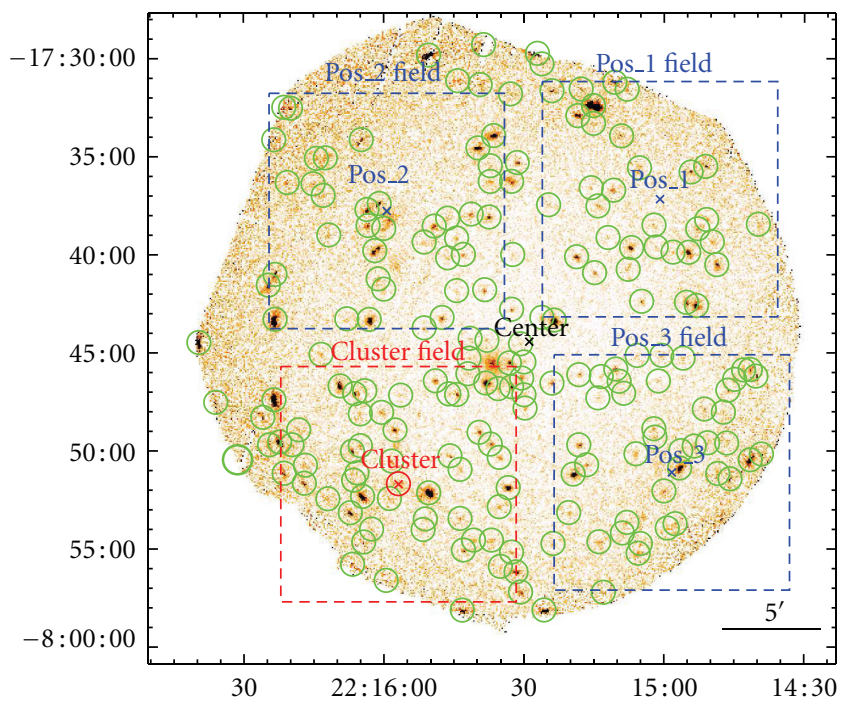

(a)

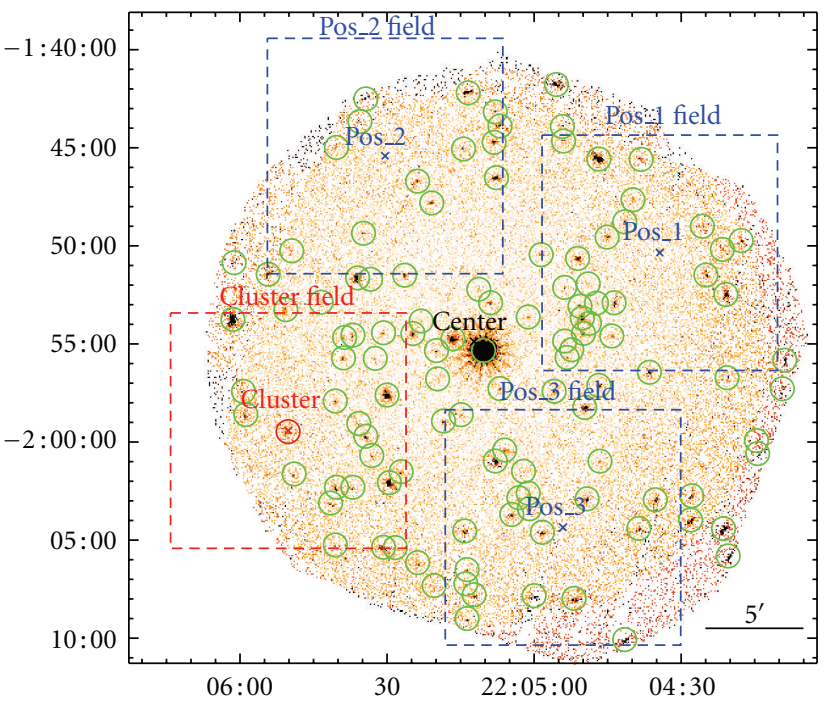

(b)

FIGURE 1: Examples of the extraction and stacking process for the $82.2 \mathrm{ksec}$ field of cluster XDCP J2215.9 - 1751 (C09) in the left panel and the $24.9 \mathrm{ksec}$ observation of the system XDCP J2205.8 - 0159 (C12) in the right panel. The cluster positions and the $12^{\prime} \times 12^{\prime}$ region around them are indicated in red. The three control fields at equivalent off-axis angles from the center position (black) are marked in blue. Green circles mark the positions of all detected soft-band X-ray point sources in the fields.

TABle 1: List of the 22 distant galaxy clusters at $z>0.9$ from Fassbender et al. [14] used for the stacking analysis in this work. The table lists a cluster identification number (column 1), the system redshift (2), the official cluster name (3), X-ray centroid coordinates $(4+5)$, the observation identification number of the XMM-Newton field used (6), the corresponding effective clean time (ECT) of the field in (7), and relevant literature references to the cluster in (8).

\begin{tabular}{|c|c|c|c|c|c|c|c|}
\hline $\begin{array}{l}\text { ID } \\
(1)\end{array}$ & $\begin{array}{c}z \\
(2) \\
\end{array}$ & $\begin{array}{c}\text { Official name } \\
\text { (3) }\end{array}$ & $\begin{array}{c}\text { RA J2000 } \\
(4)\end{array}$ & $\begin{array}{c}\text { DEC J2000 } \\
(5)\end{array}$ & $\begin{array}{l}\text { OBSID } \\
(6)\end{array}$ & $\begin{array}{c}\text { ECT ksec } \\
(7)\end{array}$ & $\begin{array}{c}\text { References } \\
(8)\end{array}$ \\
\hline $\mathrm{C} 01$ & 1.579 & XDCP J0044.0 - 2033 & $00: 44: 05.2$ & $-20: 33: 59.7$ & 0042340201 & 8.5 & {$[26]$} \\
\hline $\mathrm{C} 02$ & 1.555 & XDCP J1007.3 + 1237 & $10: 07: 21.6$ & $+12: 37: 54.3$ & 0140550601 & 19.4 & {$[27]$} \\
\hline $\mathrm{C} 03$ & 1.490 & XDCP J0338.8 + 0021 & $03: 38: 49.5$ & $+00: 21: 08.1$ & 0036540101 & 18.0 & {$[28]$} \\
\hline $\mathrm{C} 04$ & 1.457 & XMMXCS J2215.9 - 1738 & $22: 15: 58.5$ & $-17: 38: 05.8$ & 0106660101 & 51.7 & {$[10,29-31]$} \\
\hline $\mathrm{C} 05$ & 1.396 & XDCP J2235.3 - 2557 & $22: 35: 20.4$ & $-25: 57: 43.2$ & 0111790101 & 13.6 & {$[21,32]$} \\
\hline $\mathrm{C} 06$ & 1.358 & XDCP J1532.2 - 0837 & $15: 32: 13.2$ & $-08: 37: 01.4$ & 0100240801 & 22.4 & [33] \\
\hline $\mathrm{C} 07$ & 1.335 & SpARCS J0035.8 - 4312 & $00: 35: 50.1$ & $-43: 12: 10.3$ & 0148960101 & 47.2 & {$[14,34]$} \\
\hline C08 & 1.237 & RDCS J1252.9 - 2927 & $12: 52: 54.5$ & $-29: 27: 18.0$ & 0057740401 & 62.0 & [22] \\
\hline C09 & 1.227 & XDCP J2215.9 - 1751 & $22: 15: 56.9$ & $-17: 51: 40.9$ & 0106660601 & 82.2 & {$[35]$} \\
\hline $\mathrm{C} 10$ & 1.185 & XDCP J0302.1 - 0001 & $03: 02: 11.9$ & $-00: 01: 34.3$ & 0041170101 & 40.9 & {$[33]$} \\
\hline $\mathrm{C} 11$ & 1.122 & XDCP J2217.3 + 1417 & $22: 17: 20.8$ & $+14: 17: 54.6$ & 0103660301 & 10.3 & {$[14]$} \\
\hline $\mathrm{C} 12$ & 1.117 & XDCP J2205.8 - 0159 & $22: 05: 50.3$ & $-01: 59: 27.4$ & 0012440301 & 24.9 & {$[14,36]$} \\
\hline $\mathrm{C} 13$ & 1.097 & XDCP J0338.7 + 0030 & $03: 38: 44.2$ & $+00: 30: 01.8$ & 0036540101 & 18.0 & [37] \\
\hline $\mathrm{C} 14$ & 1.082 & XDCP J1007.8 + 1258 & $10: 07: 50.5$ & $+12: 58: 18.1$ & 0140550601 & 19.4 & {$[38]$} \\
\hline $\mathrm{C} 15$ & 1.053 & XLSS J0227.1 - 0418 & $02: 27: 09.2$ & $-04: 18: 00.9$ & 0112680101 & 22.7 & {$[39,40]$} \\
\hline $\mathrm{C} 16$ & 1.050 & XLSS J0224.0 - 0413 & $02: 24: 04.1$ & $-04: 13: 31.7$ & 0112680301 & 19.2 & {$[23,39,40]$} \\
\hline $\mathrm{C} 17$ & 1.000 & XDCP J2215.9 - 1740 & $22: 15: 57.5$ & $-17: 40: 25.6$ & 0106660101 & 51.7 & {$[35]$} \\
\hline $\mathrm{C} 18$ & 0.975 & XDCP J1229.4 + 0151 & $12: 29: 29.2$ & $+01: 51: 31.6$ & 0126700201 & 8.7 & {$[41]$} \\
\hline $\mathrm{C} 19$ & 0.975 & XDCP J1230.2 + 1339 & $12: 30: 16.9$ & $+13: 39: 04.3$ & 0112552101 & 10.3 & {$[18]$} \\
\hline $\mathrm{C} 20$ & 0.959 & XDCP J0027.2 + 1714 & $00: 27: 14.3$ & $+17: 14: 36.3$ & 0050140201 & 41.8 & {$[14]$} \\
\hline $\mathrm{C} 21$ & 0.947 & XDCP J0104.3 - 0630 & $01: 04: 22.3$ & $-06: 30: 03.1$ & 0112650401 & 18.4 & {$[42]$} \\
\hline $\mathrm{C} 22$ & 0.916 & XDCP J0338.5 + 0029 & $03: 38: 30.5$ & $+00: 29: 20.2$ & 0036540101 & 18.0 & {$[14]$} \\
\hline
\end{tabular}




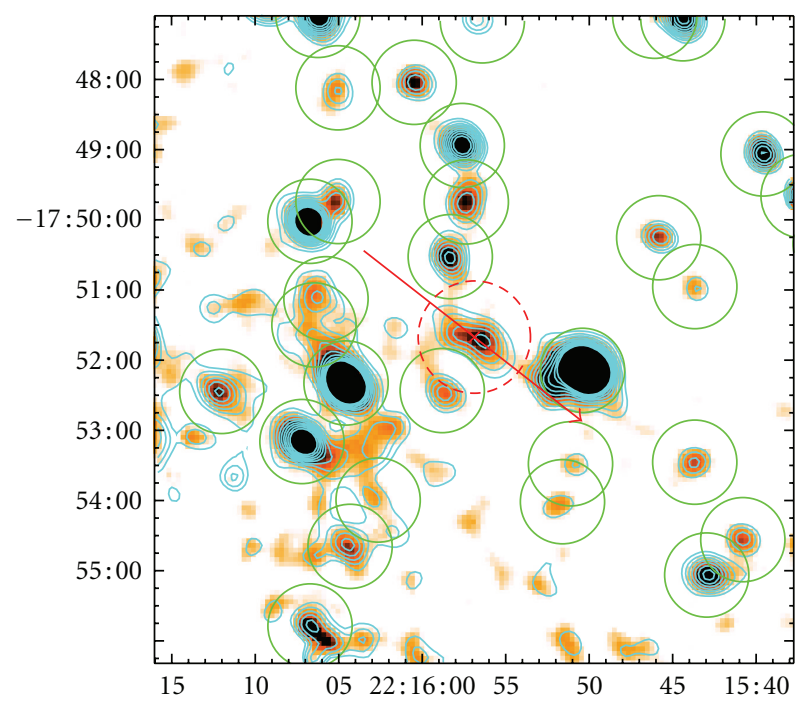

(a)

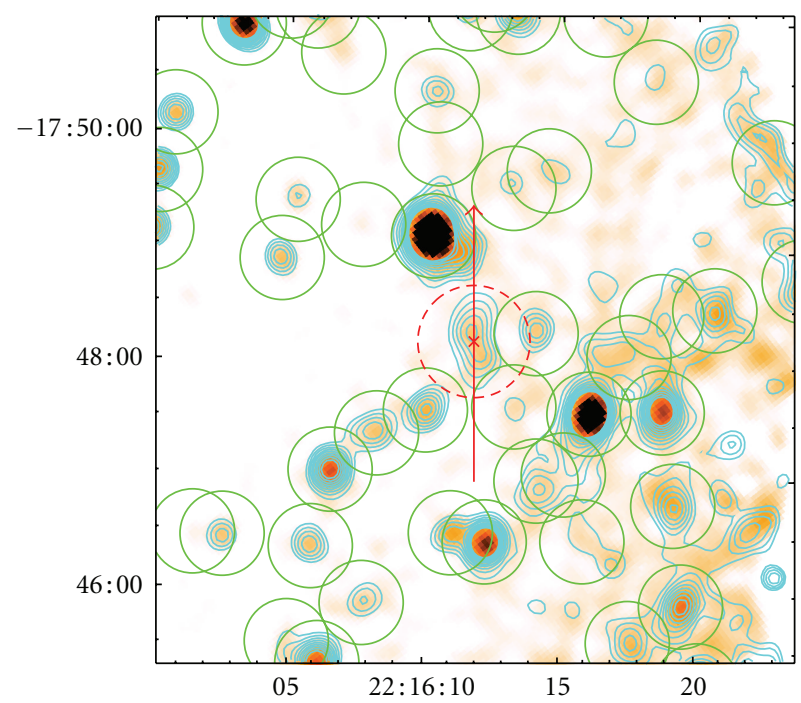

(c)

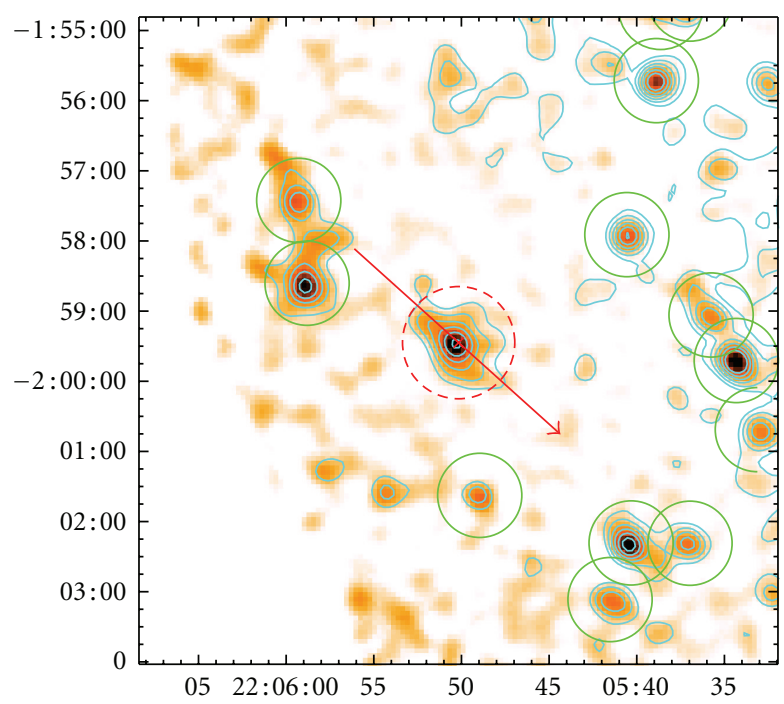

(b)

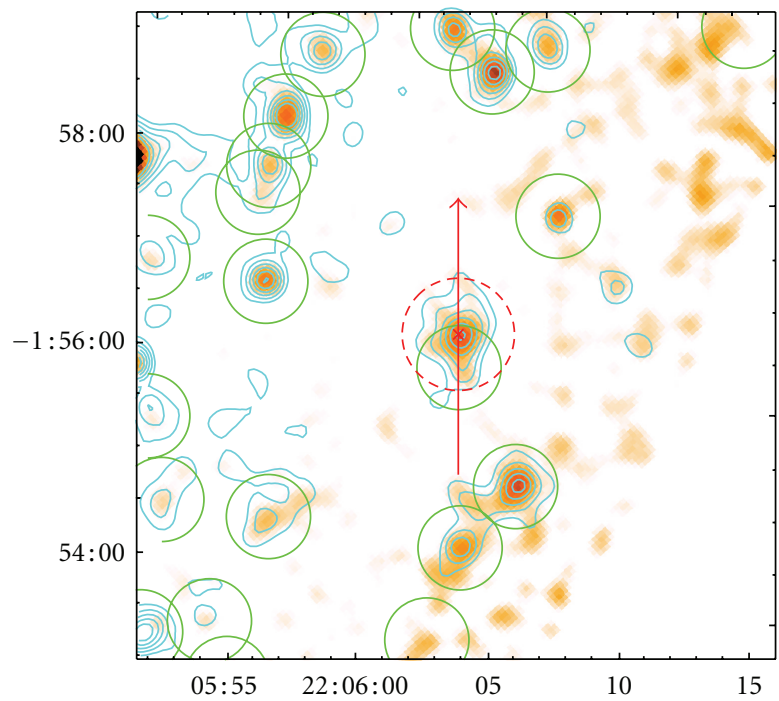

(d)

FIGURE 2: Close-up examples of the stacking process for the $9.2^{\prime} \times 9.2^{\prime}$ cluster environments of XDCP J2215.9 - 1751 (C09) in the left panels and XDCP J2205.8 - 0159 (C12) in the right panels. Cluster positions are marked by red crosses and dashed circles, and the main elongation axis of the ICM emission is indicated by the red arrow with $2^{\prime}$ length in both directions from the cluster centers. The top panels show the smoothed soft-band image and the $0.35-2.4 \mathrm{keV}$ detected sources (green circles) in their original orientation (North up, East to the left). The bottom panels display the rotated images and detected sources in the full $0.3-7.5 \mathrm{keV}$ energy range with rotation angles that align the cluster elongation axis in the North-South direction. All panels show overlaid the soft-band X-ray surface brightness contours in cyan.

were produced with the XMM Science Analysis Software (http://xmm.esac.esa.int/sas/) (SAS) tasks eboxdetect for a first sliding box detection of candidate sources followed by a maximum likelihood fitting procedure with emldetect for the final source parameter determination. For this work, the source lists of two different detection procedures are used: (1) a soft-band source detection in the energy band $0.35-2.4 \mathrm{keV}$ down to point source significances of about $3 \sigma$ and (2) a fullband detection in multiple bands covering the energy range $0.3-7.5 \mathrm{keV}$ down to point source significances of about $2 \sigma$. Although originally developed and optimized for distant cluster detections, the two schemes are able to distinguish and probe different aspects of the AGN-cluster connection. The investigation in the soft band is mostly sensitive to lowabsorption type-I AGN and can probe the effects of clusterAGN on the detection efficiency of distant clusters at lower spatial resolution with the upcoming all-sky survey eROSITA [17] as discussed in Section 4.5. The full-band detection, on the other hand, is sensitive to the full type-I and type-II AGN population and can hence provide a more complete census of AGN activity in the vicinity of distant clusters.

In order to allow a robust determination of the pure point source excess, all 105 detected extended X-ray sources in the considered fields were removed from the master source 
lists, including all of the 22 distant cluster targets listed in Table 1. Additionally, the soft- and full-band master lists were manually cleaned from obvious spurious detections $(\simeq 5.5 \%)$ in the immediate vicinity of very bright $X$-ray sources in the FoV, such as the central source displayed in the right panel of Figure 1. The final combined clean X-ray point source lists contain 2770 objects for the soft-band and 4228 sources for the full-band detection. With an effective total X-ray coverage of $4.246 \mathrm{deg}^{2}$ for the considered fields, the average point source surface densities amount to $0.182 \mathrm{srcs} / \mathrm{arcmin}^{2}$ in the soft band and $0.277 \mathrm{srcs} / \mathrm{arcmin}^{2}$ in the full band.

2.3. Point Source Stacking Analysis. In order to keep the analysis simple, the focus is placed on a possible detectable AGN point source excess in cluster environments with respect to control fields, irrespective of the flux and luminosity distribution of such sources. Owing to the varying effective exposure time of the different XMM-Newton fields in Table 1 (columns $6+7$ ), the point source detection sensitivities scale with roughly the inverse square root of the exposure time and vary across the $\mathrm{FoV}$ as a function of off-axis angle due to increased vignetting effects at larger $\Theta_{\text {off }}$. The typical $0.5-2.0 \mathrm{keV}$ soft-band point source sensitivities of $\simeq(1-2) \times 10^{-15} \mathrm{erg} \mathrm{s}^{-1} \mathrm{~cm}^{-2}$ ensure, however, that any detected point source excess associated with clusters in the redshift regime at $0.9<z \lesssim 1.6$ can be safely attributed to X-ray AGN (under the well-justified assumption that a statistically significant, background-subtracted population of excess counts is physically associated with the LSS cluster environments at the respective redshifts), rather than, for example, star-forming galaxies at lower X-ray luminosities.

For the point source stacking analysis the following approach is adapted. (a) For each of the 22 distant clusters in the considered sample a subimage is extracted from the corresponding XMM-Newton observation that places the cluster at the central image coordinates $\left(X_{\text {cen,cl }}, Y_{\text {cen,cl }}\right)$ as displayed in red in Figure 1. The same procedure is repeated at the three control field positions at rotation angles of 90 , 180 , and 270 degrees about the optical axis shown in blue in the same figure. (b) In order to ensure a homogeneous effective exposure time distribution in the final stacked cluster and control fields, the identical sub-images at the four positions as in the first step are extracted from the associated exposure maps. (c) The X-ray source lists for the soft-band and full-band detections are loaded to each of the four associated extracted subframes (one cluster plus three control positions) in the world coordinate system (WCS) and resaved in the image coordinate system of each frame. (d) For each of the four positions per cluster, the 22 extracted sub-images and exposure maps around the cluster position and control fields are coadded to result in the final deep image and exposure map stacks for each position. (e) Similarly, the 22 source lists in image coordinates at each position are concatenated into single-master-source files at each cluster and control field position for the soft-band and full-band detections separately. The source distribution of these stacked master files can now be further analyzed as a function of distance to the central extraction position with image coordinates $\left(X_{\text {cen,cl }}, Y_{\text {cen,cl }}\right)$.

The extraction procedure with the cluster (or control field) position in the center of the sub-image is depicted in the top panels of Figure 2 for the two systems C09 (left) and C12 (right). The detected extended X-ray emission of the cluster (red dashed circles) does by definition not contribute to the point source statistics (green circles). In order to also investigate potentially preferred directions of the AGN excess in cluster environments, a second variant of the previously described stacking analysis is performed. This time the visually determined main elongation axis of the detected cluster emission (red arrows in Figure 2) is assumed to be a proxy for the main matter infall and assembly axis of the cluster (e.g., see [18]). All extracted sub-images and source lists of steps $(\mathrm{a}-\mathrm{c})$ are then rotated about the central position with coordinates $\left(X_{\text {cen,cl }}, Y_{\text {cen,cl }}\right)$ in a way to align the cluster elongation axis for each of the 22 cluster fields in the Northsouth direction as shown in the lower panels of Figure 2. Subsequently, step (e) is repeated with these rotated source lists to produce a stacked master catalog of X-ray sources in a coordinate system where the cluster elongation axes are coaligned in the vertical direction.

A robust determination of the background number counts in the three control fields is of critical importance for the results of the stacking analysis. As discussed, some control field positions had to be placed at slightly larger off-axis angles compared to the cluster in order to avoid a significant overlap of the source lists at relatively small off-center distances (i.e., $<8^{\prime}$ ). Such a shift was applied to three of the 22 fields with up to $2^{\prime}$, to four fields with $2^{\prime}-4^{\prime}$, and to two fields with $>4^{\prime}$, which inevitably results in slight differences of the stacked effective clean exposure time in the cluster and control fields. Figure 3 shows these stacked exposure time profiles (left panel), from which a median fractional exposure difference for the cluster field of $d t_{\exp } / t_{\exp }=0.113=11.3 \%$ is determined as displayed by the blue dashed line in the right panel. As an important crosscheck, it can be confirmed that no significant systematic radial trend is present in the control field exposure time ratios (black line) with respect to the median.

For the deep, background-limited XMM-Newton observations this translates into an average fractional difference of the flux limit of $d f_{\text {lim }} / f_{\text {lim }} \simeq-\left(d t_{\exp } / t_{\exp }\right)^{0.5} \simeq-3.4 \%$. With the knowledge of the $\log N-\log S$ distribution from point source number counts in deep fields, the effect of the slightly increased sensitivity in the cluster field can be quantified. Using the measured faint end slope of the $\log N$ $\log S$ distribution of $\alpha=(1.65 \pm 0.05)$ [19], the fractional effect on the number counts can be determined as $d N / N \simeq$ $-(\alpha-1)(d S / S) \simeq 0.022 \simeq 2.2 \%$. For a fair evaluation of excess counts in the cluster environment, the control field counts have thus to be scaled up by the small correction factor $b_{\text {cor }} \simeq 1.022$ that accounts for the slight average sensitivity differences.

The sensitivity-corrected radial distribution of the background counts in the three control fields is displayed in Figure 4 (dotted lines) for the soft-band (top panel) and 


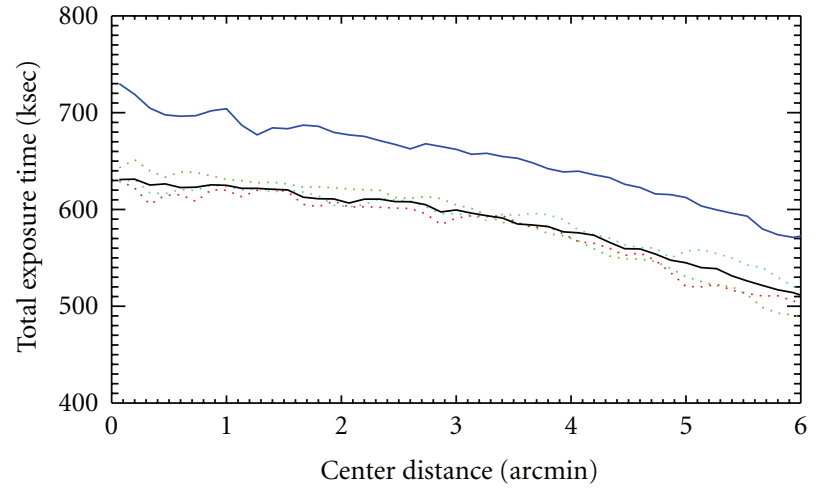

(a)

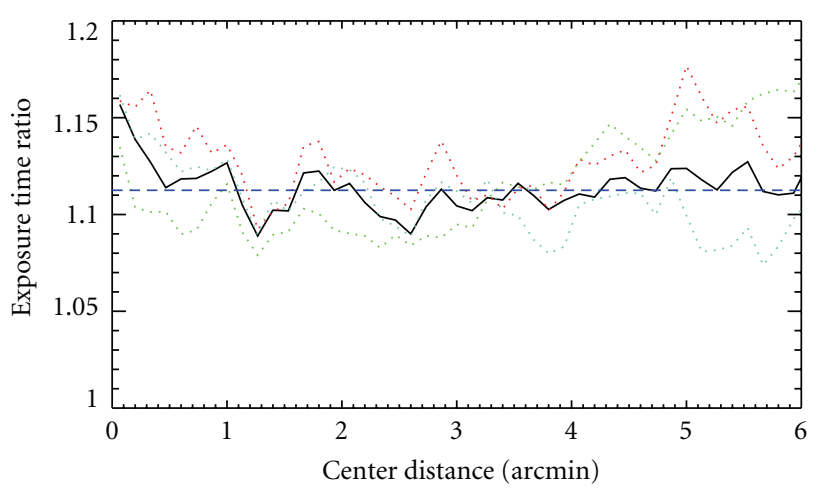

(b)

FIgURE 3: (a) Comparison of the stacked effective exposure time radial profile with respect to the defined image center positions in the cluster fields (blue solid line), the average control field (black solid line), and the three individual control fields (dotted lines). (b) Fractional difference of the stacked effective exposure time profile of the cluster field and the average control fields as a function of radial distance from the field centers (black solid line). The blue dashed line indicates the median exposure time difference of $11.3 \%$, dotted lines depict the individual control fields as before.

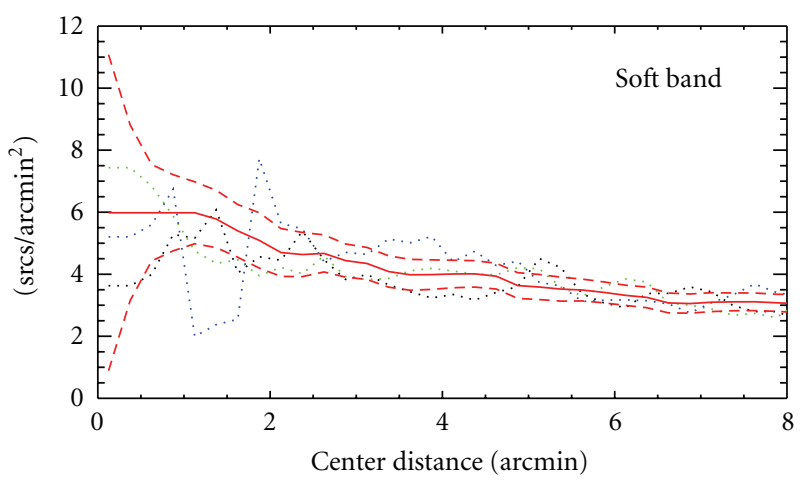

(a)

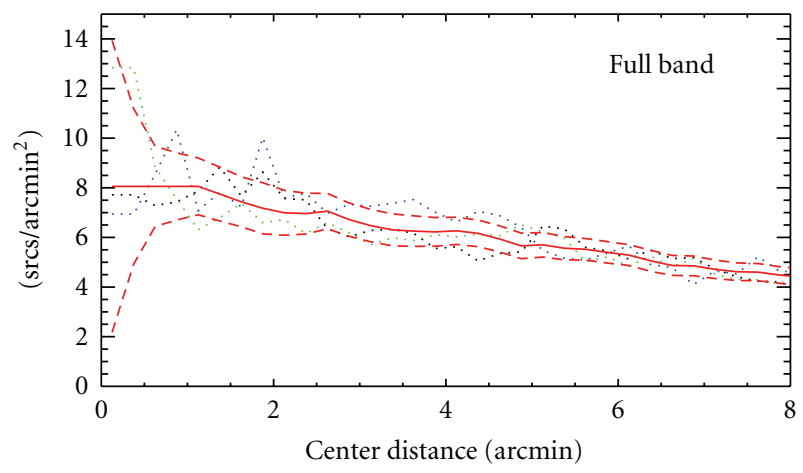

(b)

FIGURE 4: Background model as a function of center distance for the detected sources in the soft band (a) and the full band (b). The red solid line shows the average smoothed background model with $1 \sigma$ uncertainties indicated by the red dashed lines, whereas the colored dotted lines show the extracted counts in the three control fields. The gradual decline as a function of off-center angle reflects the radial change in effective exposure time as shown in Figure 3.

full-band detection (bottom panel). The final robust background model for the further analysis should on one hand reflect the gradual radial change in effective exposure time and on the other hand be a smooth function in order to suppress Poisson fluctuations in the individual radial bins, in particular close to the central position where the counts per bin drop to $\lesssim 5$. This is achieved by applying a boxcar filter with a radius of four bins (each $15^{\prime \prime}$ ) and inverse variance weighting to the averaged radial count distribution of the control fields. The resulting final average background models for the radial distribution in the soft band and full band are shown by the solid red lines in Figure 4, whereas the dashed red lines illustrate the estimated $1 \sigma$ Poisson uncertainties.

The determined background model may still be subject to residual systematic uncertainties not fully captured by the adopted approach with the three independent control fields and the applied average correction factor. Such residual systematic uncertainties include (i) slight effective exposure time offsets dependent on the exact locations of the optical axes of the different detectors, (ii) the effects of the removed extended and spurious sources, and (iii) slight geometric area mismatches at large off-center positions due to the non-axis-symmetric field-of-view edge. The magnitude of these potential systematic effects needs to be estimated for the combined stacked fields, which are the sums of the 22 random center positions across the XMM-Newton FoV. The potential exposure time offsets (i) can be shown to amount to a negligible sub-per-cent effect. Similarly, the effect of the area covered by the removed extended sources (ii) is insignificant and stacking analysis tests without any sources removed showed the qualitative same results. The largest residual systematic arises from geometric edge effects (iii), which can be estimated analogous to the statistical spatial uncertainty of a one-dimensional random walk with 22 steps, since the edge effects are randomly positive or negative relative to the cluster field. In this case, the step size for 


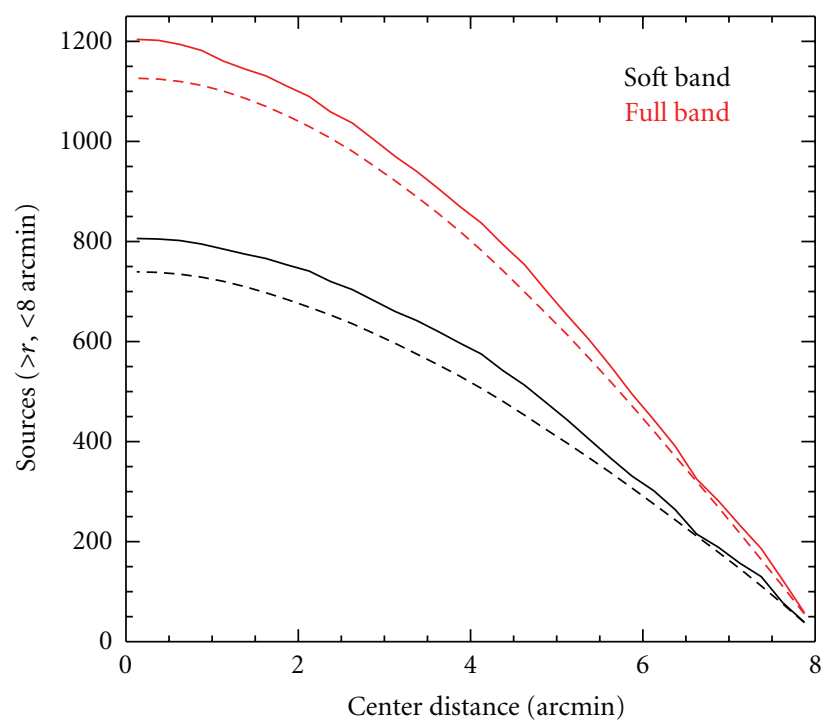

(a)

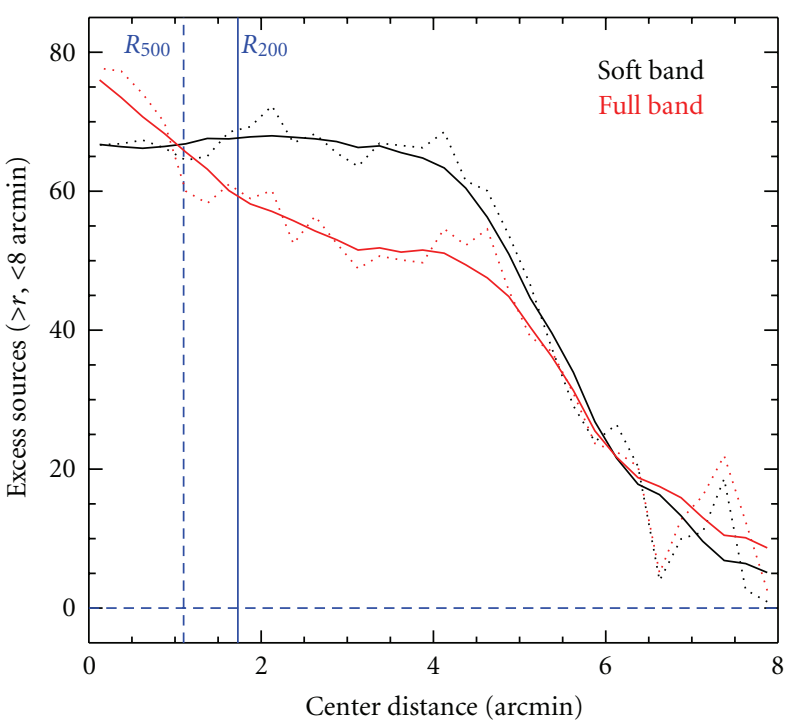

(b)

Figure 5: Cumulative number count distributions starting from an off-center angle of $8^{\prime}$ inwards. (a) Total cumulative counts for cluster fields (solid lines) in comparison to the median background models (dashed lines) for the soft-band (black) and full-band detection (red). (b) Cumulative distribution of excess counts after subtraction of the background model for the soft band (black) and full-band (red). Dotted lines show the original measured distribution, whereas solid lines depict the smoothed trends after boxcar filtering. Average cluster radii $R_{500}$ (dashed) and $R_{200}$ (solid) for the distant cluster sample are marked by the blue vertical lines.

the random walk is given by the average geometric mismatch of the analysis area per field of $8-9 \operatorname{arcmin}^{2}$, resulting in a total statistical geometric mismatch after 22 steps of $\pm \sqrt{22} \times$ $9 \simeq \pm 42 \mathrm{arcmin}^{2}$. Using the average source densities yields approximate upper limits for the total impact of this effect of $\sigma_{\text {sys }}($ full-band $) \lesssim 12$ sources and $\sigma_{\text {sys }}($ soft-band $) \lesssim 7$ point sources. Consequently, the total systematic error budget is estimated to be $\lesssim 60 \%$ of the total Poisson uncertainties derived in the next section and is hence still subdominant compared to the statistical errors.

\section{Results}

In the following, the results of the X-ray stacking analysis are presented for the cumulative number counts, the radial distribution of excess counts, and an evaluation of source counts along the principal cluster elongation axis. All results are given in stacked units, that is, the sum of all 22 cluster environments is combined into a single radial X-ray source count profile. For all analyses a radial bin size for each independent ring segment of $15^{\prime \prime}$ is used and the maximal off-center radius is limited to $8^{\prime}(\sim 4 \mathrm{Mpc})$ in order to avoid significant field-of-view edge effects.

3.1. Cumulative Source Counts. The left panel of Figure 5 displays the cumulative number counts for the cluster (red and black) and average background field counts (blue) starting at a radial distance of $8^{\prime}$ and moving inwards towards the center position. A significant excess of X-ray sources in the cluster field is apparent starting at off-center distances of about $6^{\prime}$ in both the soft-band (lower dashed lines) and full-band counts (upper solid lines). For the full-band source list the number of excess X-ray counts in the cluster field within the $8^{\prime}$ analysis radius is about 78 , while the soft band shows approximately 67 excess counts. This translates into a fractional source excess in the distant cluster environments of $+9.0 \%$ in the soft band and $+6.9 \%$ in the full band.

Interestingly, the radial distribution of the excess counts for the two different band schemes is different, which is shown in the right panel of Figure 5. The smoothed trends (solid lines) indicate that the main excess counts in the softband (black) originate at cluster-centric distance of 4-6', corresponding to $2-3 \mathrm{Mpc}$, while excess source counts in the full band rise sharply all the way to the center.

Applying Poisson statistics to the total cumulative background counts, which are based on three independent control fields, the statistical $1 \sigma$ uncertainties can be estimated as $\sqrt{N_{\text {tot }} / 3}$, which amounts to $\sigma_{\text {tot,stat }}^{\text {soft }} \simeq 15.7$ sources and $\sigma_{\text {tot } \text {,stat }}^{\text {full }} \simeq 19.4$ counts for the two different band schemes. The significance of the total detected X-ray source excess within a cluster-centric distance of $8^{\prime}$ is hence $4.2 \sigma_{\text {stat }}$ in the soft-band and $4.0 \sigma_{\text {stat }}$ for the full-band detection. The average number of detected excess X-ray AGN per cluster environment amounts to $3.0 \pm 0.7$ softband and $3.5 \pm 0.9$ fullband AGN, respectively, which are to be interpreted as lower limits since not the full geometric area out to $8^{\prime}$ was covered in the detector FoV for each cluster environment.

3.2. Radial Distribution of Excess AGN. The backgroundsubtracted radial distribution of excess X-ray sources in Figure 6 shows the data points for each individual bin with Poisson errors (black) and the smoothed radial trend 


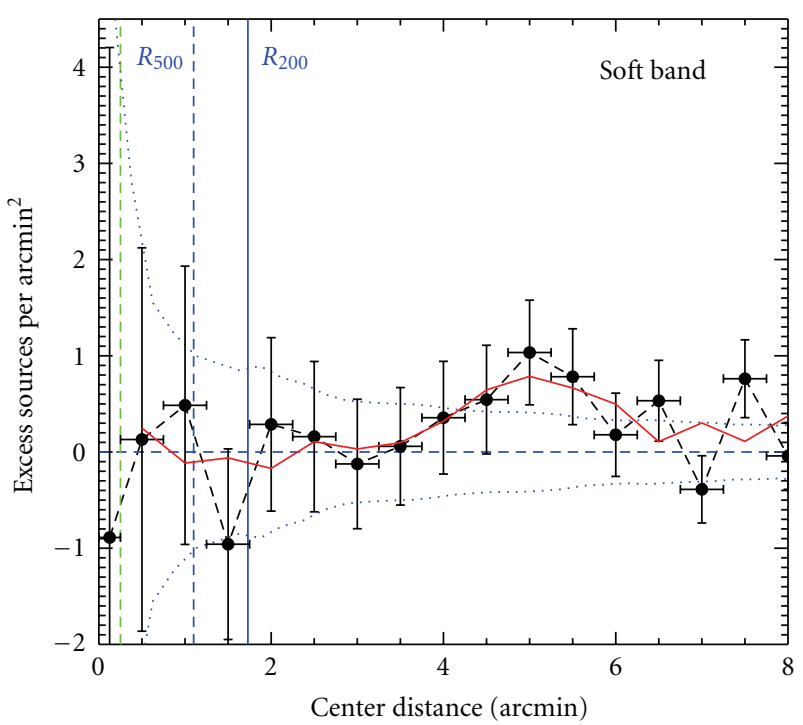

(a)

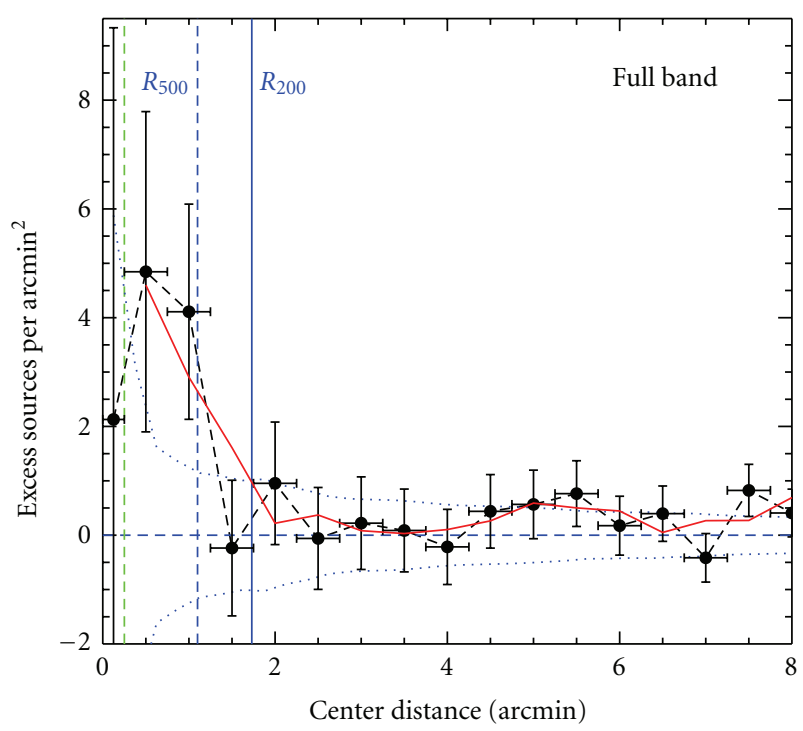

(b)

FIgURE 6: Background-subtracted radial distributions of excess X-ray sources for the soft band (a) and full-band (b). Dotted blue lines indicate the $1 \sigma$ uncertainties about the mean background counts (blue dashed). Data points and the black dashed line display the measurements in each of the independent radial bins, whereas the red solid line shows the boxcar smoothed radial trend. The green dashed vertical line on the left shows the $15^{\prime \prime}$ radius below which the measurements are biased due to the comparable spatial resolution of XMM-Newton. The blue vertical lines mark the average cluster radii $R_{500}$ (dashed) and $R_{200}$ (solid).

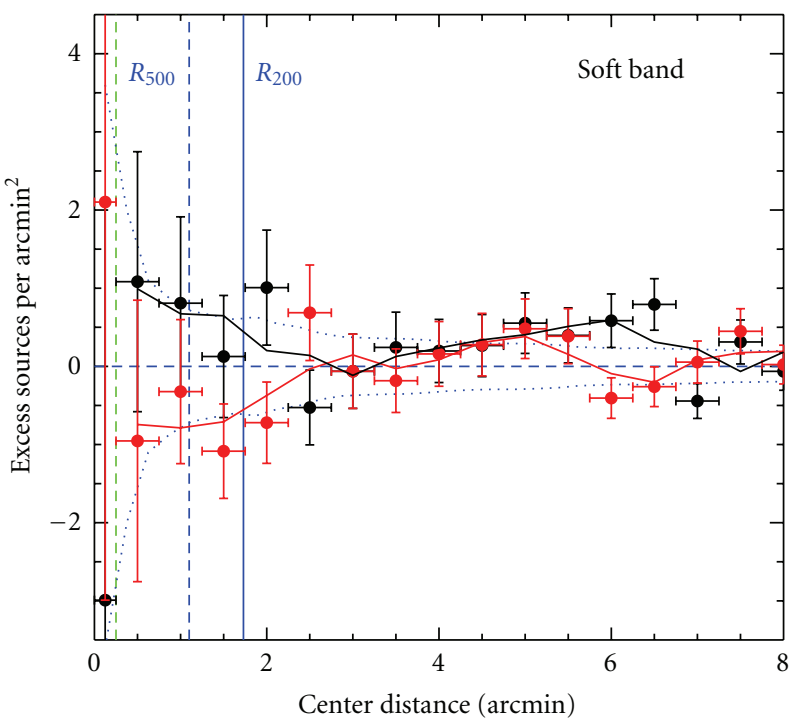

(a)

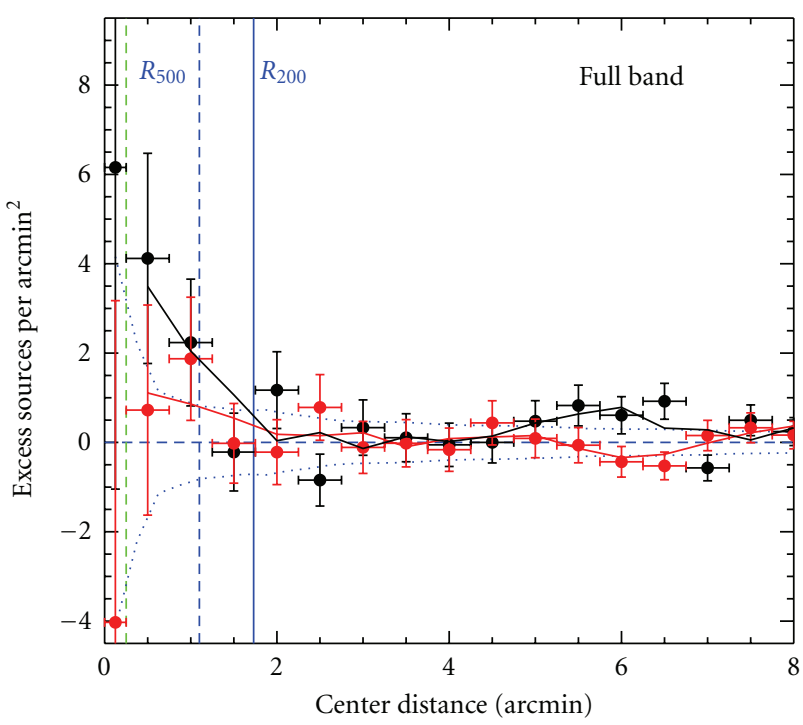

(b)

FIGURE 7: Same as Figure 6 but now distinguishing the sectors aligned with the cluster elongations (black points and solid black line) and the perpendicular direction (red points and solid red line).

(solid red line) for the soft-band (upper panel) and fullband detection (lower panel). The observed trends in the cumulative distribution of the previous section can now be evaluated for the independent data points with associated error bars and the given uncertainty in the subtracted background counts (blue dotted lines). For representation purposes (Figures 6 and 7) and better statistics in each bin, the displayed radial bins were increased to $30^{\prime \prime}$ each outside the biased central 30" region (green dashed vertical line, (see Section 2.1)), which is also excluded for the smoothed solid trend lines.

In the soft band (upper panel), the most significant feature in the radial distribution is the hump between clustercentric distance $4^{\prime}-6^{\prime}$ as depicted by the red trend line. The covered solid angle at cluster-centric distances $4^{\prime} \leq r \leq 6^{\prime}$ is 63 square arcmin implying that significant excess counts 
normalized to a unit area are hard to measure in comparison to the background counts. However, in this $4^{\prime} \lesssim r \lesssim 6^{\prime}$ range six independent adjacent radial bins show an excess, of which four have more than $1 \sigma$ significance each, yielding a high combined confidence that this feature is indeed real. The hump is also discernible in the full-band detection (lower panel), although at lower statistical significance.

The most prominent feature in the full-band detection is the strong rise of excess X-ray sources at radial distances below $2^{\prime}$, which correspond approximately to the radius inside the fiducial $R_{200}$ (vertical blue line) of the distant cluster sample. $\left(R_{200}\left(R_{500}\right)\right.$ are the radii for which the mean enclosed total mass density of the cluster is 200 (500) times the critical energy density of the Universe $\rho_{\text {cr }}(z)$ at the given redshift $z$. The considered distant cluster sample has an average $R_{200}\left(R_{500}\right)$ of about $100^{\prime \prime}\left(64^{\prime \prime}\right)$ with a standard deviation of $\pm 20 \%$ ). Owing to the relatively small enclosed central area with a corresponding low number of total counts, the statistical uncertainties inside the fiducial cluster regions are quite significant. Nevertheless, the two innermost bins outside the core region indicate a clear and significant trend of a steep inner radial profile of full-band detected excess sources. The inner profile of the soft-band sources in the upper panel, on the other hand, is fully consistent with a null excess of sources inside radii $r<2^{\prime}$.

\subsection{X-Ray Counts along the Principal Cluster Elongation Axis.} In order to investigate the possibility that AGN activity occurs along a preferential direction in the cluster-frame system, the radial profile analysis for excess X-ray counts was repeated with the rotated stacked source lists that aligned the principal elongation axis of the cluster X-ray emission in the vertical direction (see Section 2.3 and bottom panels of Figure 2). The radial distribution was then split into two disjoint sectors, the aligned sector along the cluster elongation direction covering the range within an angle of $\pm 45^{\circ}$ to the vertical axis with the cluster center as origin and the perpendicular sector within $\pm 45^{\circ}$ to the horizontal coordinate axis. The average background models (Figure 4) and the associated uncertainties were adopted to account for the factor of two smaller total area covered by each of the two sectors. The background subtracted radial counts along the aligned (black) and perpendicular sectors (red) are displayed in Figure 7 for the soft-band (upper panel) and full-band detection (lower panel).

For the full-band counts in the lower panel the profiles at $r<2^{\prime}$ indicate that the X-ray source excess inside the fiducial cluster radius $R_{200}$ is dominated by AGN along the clusteraligned direction (black line). Additionally, the secondary hump at $4.5^{\prime}-6.5^{\prime}$ is now more significant in the aligned (black) direction, while consistent with a null excess for the perpendicular sector (red). However, owing to the lower number of excess counts in the individual split sector bins with a corresponding decreased statistical significance these results are to be considered as tentative.

For the soft-band detection in the upper panel, the $4^{\prime}-$ $6^{\prime}$ hump appears also to be enhanced in the aligned (black) direction around $r \sim 6^{\prime}$. Below a radius of $2^{\prime}$ the sector counts seem to split into an excess in the aligned and a deficit in the perpendicular sector, but both are on the level of the background uncertainty.

3.4. Robustness of Results. The tentative nature of the latter results in Section 3.3 with the separated sectors along two directions illustrates the limits of the statistical detectability of radial features in the X-ray source distribution for the currently available sample size of distant X-ray clusters. However, the results based on the overall excess counts in the radial distributions in Sections 3.1 and 3.2 are detected on the $4 \sigma$ level (for $r<8^{\prime}$ ) and can hence be considered as robust.

In particular the central rise of the full-band counts and the soft-band $4^{\prime}-6^{\prime}$ hump seem to be real features in the radial distribution of distant cluster environments with an average of 1-2 excess sources per cluster in each feature. A central rise in the full band while flat in the soft band could well be physically explained by a dominance of faint and spectrally hard cluster AGN. With on average about one full-band excess source per cluster in the inner $\sim 2^{\prime}$, the central part of the background model would have to be underestimated by almost a factor of two to attribute the observed cumulative excess to a background systematic.

The cumulative counts for the $4^{\prime}-6^{\prime}$ hump in the soft band exceed the full-band counts by about 15 sources around $r \simeq 4^{\prime}$ (see right panel of Figure 5). On the other hand, all soft-band sources should also be part of the full-band catalogs, that is, the cumulative full-band excess would be expected to be at least as high for the soft-band. However, this slight discrepancy at intermediate off-center angles between $2^{\prime}$ and $5^{\prime}$ in the cumulative excess distribution can likely be attributed to Poisson noise in the full-band background counts, which was estimated to be slightly higher in magnitude compared to the measured difference of excess soft-band sources at the hump location. The full-band signal at these intermediate off-center distances hence seems to be diluted by the larger Poisson noise at a level within the statistical expectations.

The presence of the hump in the full band itself, although at lower confidence level, provides some extra confidence in its real nature. Other cross-checks with the exposure time profile (Figure 3 ) and the background (Figure 4) do not reveal any particular radial feature in the $4^{\prime}-6^{\prime}$ range that could attribute the observed hump to a systematic. The combined systematic error budget was estimated in Section 2.3 to be at most $60 \%$ of the total statistical Poisson error ( $\lesssim 12$ sources), which is far below the observed signal. The cumulative excess magnitude in the hump of more than three dozen sources also makes the possibility of individual systematic structures at $\sim 5^{\prime}$ in the cluster fields an unlikely explanation.

\section{Discussion}

4.1. Spectroscopically Confirmed AGN. The ultimate proof to quantify the statistical properties of AGN in high- $z$ clusters from the centers to the large-scale environments would 
TABLE 2: List of spectroscopically confirmed X-ray AGN within a search radius of $12^{\prime}(\simeq 6 \mathrm{Mpc})$ from the cluster centers and with a spectroscopic redshift that is within $\pm 3000 \mathrm{~km} / \mathrm{s}$ of the cluster restframe velocity. The table lists the cluster environment ID of Table 1 in column (1), an AGN ID in (2), the angular distance to the cluster center in (3), the AGN redshift in (4), the RA and DEC coordinates in (5-6), the source name as listed in NED in (7), and a redshift reference in (8).

\begin{tabular}{|c|c|c|c|c|c|c|c|}
\hline $\begin{array}{l}\text { Cl. env. } \\
\text { (1) }\end{array}$ & $\begin{array}{l}\text { AGN ID } \\
(2)\end{array}$ & $\begin{array}{c}\text { Distance arcmin } \\
\text { (3) }\end{array}$ & $\begin{array}{c}z \\
(4)\end{array}$ & $\begin{array}{c}\text { RA J2000 } \\
(5)\end{array}$ & $\begin{array}{c}\text { DEC J2000 } \\
(6)\end{array}$ & $\begin{array}{c}\text { NED name } \\
(7) \\
\end{array}$ & $\begin{array}{c}\text { Reference } \\
(8)\end{array}$ \\
\hline $\mathrm{C} 03$ & A01 & 9.52 & 1.486 & $03: 39: 08.3$ & $+00: 29: 25$ & SDSS J033908.27 + 002924.9 & {$[43]$} \\
\hline $\mathrm{C} 03$ & A02 & 11.3 & 1.483 & $03: 39: 12.3$ & $+00: 30: 56$ & SDSS J033912.34 + 003055.5 & {$[43]$} \\
\hline $\mathrm{C} 04$ & A03 & 1.31 & 1.462 & $22: 16: 03.7$ & $-17: 38: 31$ & XMMUJ221603.6 - 173830 & {$[44]$} \\
\hline $\mathrm{C} 04$ & A04 & 0.24 & 1.462 & $22: 15: 59.1$ & $-17: 37: 54$ & PS1 & {$[10]$} \\
\hline $\mathrm{C} 04$ & A05 & 0.12 & 1.453 & $22: 15: 58.9$ & $-17: 38: 10$ & PS2 & {$[10]$} \\
\hline $\mathrm{C} 06$ & A06 & 6.36 & 1.373 & $15: 32: 06.0$ & $-08: 30: 55$ & XMS J153206.0 - 083055 & {$[45]$} \\
\hline $\mathrm{C} 07$ & $\mathrm{~A} 07$ & 5.32 & 1.330 & $00: 36: 18.6$ & $-43: 13: 20$ & IRAC 109477 & {$[46]$} \\
\hline $\mathrm{C} 07$ & A08 & 6.52 & 1.318 & $00: 35: 23.9$ & $-43: 16: 38$ & IRAC 229193 & {$[47]$} \\
\hline $\mathrm{C} 07$ & A09 & 9.02 & 1.334 & $00: 36: 22.1$ & $-43: 19: 03$ & IRAC 111580 & {$[47]$} \\
\hline $\mathrm{C} 08$ & A10 & 7.81 & 1.234 & $12: 53: 08.3$ & $-29: 20: 10$ & GMOS-F2 08 & {$[48]$} \\
\hline $\mathrm{C} 10$ & A11 & 3.08 & 1.179 & $03: 02: 14.8$ & $+00: 01: 25$ & SDSS J030214.82+000125.3 & {$[43]$} \\
\hline $\mathrm{C} 12$ & A12 & 11.6 & 1.110 & $22: 06: 26.0$ & $-01: 52: 01$ & FBQS J2206 - 0152 & {$[49]$} \\
\hline $\mathrm{C} 13$ & A13 & 10.8 & 1.120 & $03: 38: 10.2$ & $+00: 23: 25$ & SDSS J033810.16 + 002325.1 & {$[43]$} \\
\hline $\mathrm{C} 15$ & A14 & 7.97 & 1.053 & $02: 26: 38.0$ & $-04: 19: 45$ & VVDS 020465089 & {$[50]$} \\
\hline $\mathrm{C} 21$ & A15 & 7.66 & 0.932 & $01: 04: 02.8$ & $-06: 36: 00$ & XMMUJ0104.1 - 0635000 & {$[51]$} \\
\hline
\end{tabular}

require a combination of high-resolution X-ray imaging plus an optical spectroscopic identification to identify all X-ray point sources. Such a data set is currently not yet available for sizable samples of $z>0.9 \mathrm{X}$-ray luminous systems. However, as a starting point the observed Xray point source catalogs can be crossmatched with public redshift data from the NASA Extragalactic Data Base (http://nedwww.ipac.caltech.edu) (NED) complemented by individual cluster publications [10]. The resulting list of 15 spectroscopically confirmed and X-ray identified AGN within $12^{\prime}$ of the cluster positions and spectroscopic restframe velocity offsets of less than $\pm 3000 \mathrm{~km} / \mathrm{s}$ is shown in Table 2.

Five of the spectroscopic AGN are located at clustercentric angular separations $8^{\prime}<r<12^{\prime}$, corresponding to projected distances of 4-6 Mpc. Ten spectroscopic members are found at the probed radial distance range of $\leq 8^{\prime}$, four of them at $<4^{\prime}(<2 \mathrm{Mpc})$ and six in the range $4^{\prime}<r<8^{\prime}$ (2-4 Mpc). The two objects A04 and A05 at the center of XMMXCS J2215.9 - 1738 at $z=1.46$ are not spatially resolved with XMM-Newton [10]; all others are identified $\mathrm{X}$-ray point sources (except object A12, which is outside the XMM-Newton FoV, but is classified in NED as QSO).

Out of the measured full-band X-ray point source excess of about $78( \pm 25 \%)$ in the cluster environments at $r \leq 8^{\prime}$, eight or about $10 \%$ are spectroscopically confirmed $\mathrm{X}$ ray AGN at the cluster redshift. As can be expected, the serendipitous spectroscopic identification rate of cluster AGN in this high- $z$ sample is still fairly low, but sufficiently high to confirm the presence of X-ray AGN in the large-scale structure environment of the probed systems.
4.2. Selection Effects and Comparison to Chandra Observations. The considered distant galaxy cluster sample is $\mathrm{X}$ ray selected based on XMM-Newton data as discussed in Sections 2.1 and 2.2, implying that potential selection effects could have an impact on some of the results presented in Section 3. All clusters were originally detected as extended $\mathrm{X}$-ray sources, with most of the detection weight originating from the soft band owing to the expected spectral properties of high- $z$ thermal I CM emission. In principle, cluster-embedded X-ray point sources could have both an (negative) antibias or (positive) bias effect on the XMMNewton selection. An anti-bias, that is, a missed fraction of proper cluster sources, arises when a bright central AGN is dominating over the extended thermal emission in the soft band, resulting in a potential misclassification of the cluster as an X-ray point source. On the other hand, an opposite bias effect could arise in the cases where a weak unresolved cluster AGN adds a subdominant fraction of flux to the underlying extended cluster emission or where the superposition of multiple embedded X-ray point sources even mimics an Xray extended at the given XMM-Newton resolution limit.

Neither of the described possible anti-bias or bias effects are currently accessible to a robust quantitative evaluation since this would require large well-defined $z>0.9$ cluster samples based on different selection techniques (e.g., IR, SZE, and X-ray) and followed up with high-resolution Chandra observations and extensive optical spectroscopy that are not yet available for such a study. The two most distant spectroscopically confirmed infrared selected systems in the galaxy group mass regime, CL J1449+0856 at $z=$ 2.07 [20] and SXDF-XCL J0218 - 0510 at $z=1.62$ [11], 
feature both a central X-ray point source that dominates the $\mathrm{X}$-ray emission and could hint at a common occurrence of central AGN in low-mass systems at $z \gtrsim 1$.6. However, given the different system masses, redshift regime, and selection techniques such first hints based on low-number statistics may not be representative for the considered X-ray selected high- $z$ cluster sample.

A meaningful cross-check on the influence of potentially unresolved embedded cluster AGN for the cluster sample of this work can currently only be obtained from the available published Chandra observations of a subsample of the five systems C04 [10], C05 [21], C08 [22], C16 [23], and C19 [18] listed in Table 1. For the four systems at $z \lesssim 1.4$ (C05, C08, C16, C19) Chandra did not reveal any additional central point sources embedded in the extended ICM emission. Only for the system C04 at $z=1.46$ the high-resolution observations disclosed the two additional sub-dominant clusters AGN A04 and A05 (see Table 2 and Section 4.1) at $r \lesssim 15^{\prime \prime}$ that are unresolved with XMM-Newton both for the soft- and full-band detection. The cross-check with this Chandra subsample is hence consistent with a fair XMMNewton accounting of X-ray point sources in the distant cluster environments outside the biased central $15^{\prime \prime}$ radius, which was excluded from the trend analysis of the radial profiles in Sections 3.2 and 3.3. In particular, there is no indication that the soft-band radial point source profile could be antibiased in the range $0.25^{\prime}<r \lesssim 2^{\prime}$ due to the presence of extended ICM emission. Similarly, the Chandra sub-sample does not reveal any cases of systems that entered the distant X-ray cluster sample solely based on a bias effect of embedded point sources.

In summary, although some sample selection effects inherit to the XMM-Newton discovery method for the distant clusters may apply compared to an ideal (nonexistent) sample, a qualitative cross-check with the available Chandra observations for five systems did not reveal any evident biases concerning the radial profile of point sources in the main targeted cluster-centric distance range of $0.25^{\prime}<r \lesssim 8^{\prime}$.

4.3. Comparison to Previous Studies. The high- $z$ results of this work are consistent with numerous previous Chandra studies that find a general trend of increasing AGN activity in cluster environments with redshift for X-ray selected samples (e.g., $[1-3,5])$ and infrared-selected clusters [12]. Although the used sample is X-ray selected with XMM-Newton, implying the discussed natural anti-bias against potential systems with bright central soft-band AGN, a centrally rising radial distribution of lower luminosity AGN was found with about one detected excess source per system within a projected radius of $1 \mathrm{Mpc}$. However, because of the limited spatial resolution of XMM-Newton, the recovered excess sources are incomplete in the very core $\left(r \lesssim 15-20^{\prime \prime}\right)$, where individual systems at the highest accessible redshifts have revealed embedded cluster AGN as discussed in Section 4.2.

Concerning the detected secondary $4^{\prime}-6^{\prime}$ hump (2$3 \mathrm{Mpc}$ ), Ruderman and Ebeling [6] reported on the same general radial profile shape of the X-ray point source excess around very massive MACS clusters at intermediate redshifts $(0.3<z<0.7)$ with a central spike plus an additional broad excess at projected cluster-centric distances of 2-3 Mpc. They interpreted this finding as evidence for distinct triggering mechanisms of nuclear activity in the center through close encounters of infalling galaxies, and the cluster-field interface, where galaxy mergers fuel the central supermassive black holes. On the other hand, Gilmour et al. [4] did not recover the secondary radial excess feature in their larger sample of 148 clusters at $z<0.9$. Their tests with cluster subsamples revealed that a point source excess at $r>2$ $\mathrm{Mpc}$ may be systematically boosted by structures related to nonassociated foreground clusters in the FoV [4].

With respect to such a test, the current sample size of 22 high- $z$ cluster fields is still too small to discard all fields with a potential contamination from foreground structures, which are ubiquitous at the exposure depth of the considered observations. For the nature of the $4^{\prime}-6^{\prime}$ hump, a systematic bias related to foreground structures can hence not be fully ruled out at this point, although the measured excess in this region would require multiple such structures not captured by the background model. A test with the two most crowded fields removed from the analysis (excluding clusters C03, C04, C09, C13, C17, and C22) showed no qualitative difference concerning the shape and the presence of the hump.

On the other hand, a cluster-associated excess of X-ray AGN at projected cluster-centric radii of $r>4^{\prime}(>2 \mathrm{Mpc})$ must be present as revealed by the 11 spectroscopically confirmed objects in Table 2 in the range $4^{\prime}<r<12^{\prime}$. Moreover, Rumbaugh et al. [9] showed in their spectroscopic study of cluster environments in the redshift range 0.7-0.9 that half of the AGN host galaxies are located at projected distances of $>1.5 \mathrm{Mpc}$ away from the nearest cluster or group. In particular, they also confirmed a significant difference in AGN X-ray luminosity between objects in the dense inner cluster regions and the low-density large-scale structure environments in the sense that the central objects show low luminosities, while the brightest objects are all found at large cluster centric distances.

4.4. The Distant Cluster-AGN Connection. Taking the presented results at face value, the following scenario for AGN activity in distant X-ray cluster environments at $0.9<z \lesssim$ 1.6 emerges. The detected significant $(4 \sigma)$ excess of X-ray AGN in the distant cluster environments at angular radii of up to $8^{\prime}$ is split into two distinct populations that reflect different triggering mechanisms of nuclear activity.

At cluster-centric radii less than the fiducial average cluster size of the sample of $R_{200} \simeq 830 \mathrm{kpc} \simeq 1.7^{\prime}$ a centrally peaked population of low-luminosity X-ray AGN exists. The conclusion of low X-ray luminosities (or a heavily absorbed type-II spectrum) for this population originates from the fact that the central excess is not observed in the soft-band detection with its higher source significance threshold. The tentative result of Section 3.3 suggests that the AGN activity preferentially occurs along the main matter infall axis of the cluster, as indicated by the principal elongation axis of the extended cluster emission. This nuclear activity is 
likely triggered by close encounters of infalling objects and is mostly found in red or green transitional galaxies as reported in [9]. Overall, the detected (incomplete) excess of about one AGN source per cluster field inside its fiducial radius $R_{200}$ is still very moderate when considering the expected (e.g., [24]) average enclosed stellar mass of $\simeq 4 \times 10^{12} \mathrm{M}_{\odot}$ for the cluster systems. Comparing this value to the average AGN abundance in the COSMOS field in the same $0.9 \leq z \leq 1.6$ redshift range of one X-ray AGN per $2 \times 10^{12} \mathrm{M}_{\odot}$ in stellar mass (Bongiorno et al., in prep.) suggests that the AGN activity in high- $z$ cluster environments is still suppressed by a factor of two compared to the field abundance.

The second AGN population is preferentially located at cluster-centric distances of 2-3 Mpc as part of the observed $4^{\prime}-6^{\prime}$ hump of excess sources. The soft-band detection of this feature indicates significantly higher soft-band X-ray luminosities compared to the central AGN. The projected distance of the hump location corresponds to about $3 \times R_{200}$ of the average cluster radius. At this distance, the relative galaxy velocities are still small while the object density is already significantly enhanced, resulting in an environment where major merging processes are expected to be efficient. The observed AGN excess in the larger-scale environment of distant X-ray clusters is hence likely to be attributed to merger-induced nuclear activity in "quasar mode" [25] occurring at a potential sweet spot for merging events in the infall regions at cluster-centric projected distances of around $3 \times R_{200}$.

4.5. Implications for eROSITA. The next generation all-sky $\mathrm{X}$-ray survey will be conducted by the upcoming eROSITA mission [17], which has an expected average survey PSF of $25^{\prime \prime}-30^{\prime \prime}$ and hence provides a lower spatial resolution than Chandra and XMM-Newton. In order to evaluate the performance for distant cluster applications, the high- $z$ AGN excess close to the cluster position is of critical importance.

The measured average excess of about one full-banddetected AGN within $R_{200}$ with a centrally peaked radial profile may pose a severe potential bias for X-ray spectroscopic applications at $z>0.9$, most notably high- $z$ ICM temperature measurements. For faint and compact high $-z$ systems, the full detected X-ray emission within the extraction aperture has to be considered to obtain enough signal, without the possibility to subtract embedded point sources. Hidden AGN can hence harden the spectrum and bias the $T_{X}$ determination high (see, e.g., [10]).

Although the statistical uncertainties in the central region are still large, the measured soft-band radial profile did not reveal a significant central excess of AGN. The situation for the detection of extended high- $z$ Xray sources and accurate soft-band flux determinations hence still looks very promising. These tasks can be performed in the soft-band only, most commonly in the $0.5-2 \mathrm{keV}$ range, where the cluster AGN contribution seems to be low for the presented X-ray selected sample and sensitivities, which correspond to the planned eROSITA deep fields. The detectability of distant clusters with eROSITA may hence not be significantly influenced by the present findings. The measured soft-band
X-ray luminosity for high- $z$ eROSITA clusters will hence likely be the preferable low-bias mass proxy.

\section{Summary and Conclusions}

This work investigated the X-ray point source excess in the environment of distant X-ray luminous galaxy clusters in the redshift range $0.9<z \lesssim 1.6$ based on a sample of 22 systems with an average mass of $2 \times 10^{14} \mathrm{M}_{\odot}$ compiled by the XMM-Newton Distant Cluster Project. The X-ray source detection in the available XMM-Newton observations for each cluster with a median clean field exposure time of $19 \mathrm{ksec}$ was performed in two energy ranges, a $0.35-$ $2.4 \mathrm{keV}$ soft band and a $0.3-7.5 \mathrm{keV}$ full band. The X-ray source counts were stacked in cluster-centric coordinates and compared to the average background counts extracted from three independent control fields in the XMM-Newton field of view. The main findings of this $\mathrm{X}$-ray stacking analysis can be summarized as follows.

(1) The cumulative radial X-ray source counts within a cluster-centric region of $8^{\prime}$ reveal a significant excess of $\sim 67$ (softband) and $\sim 78$ (soft-band) sources with a statistical confidence of $4.2 \sigma$ in the soft band and $4.0 \sigma$ in the full band. The resulting average detected point source excess of $3.0 \pm 0.7$ (soft-band) and $3.5 \pm$ 0.9 (full-band) sources per cluster environment is to be interpreted as lower limit due to an incomplete coverage of the full geometric area out to $8^{\prime}$.

(2) The radial cumulative distributions of the detected excess counts show a different radial behavior for the soft band and full band. Soft-band detected excess sources are mainly located at cluster-centric projected distances of $2-3 \mathrm{Mpc}$ (the $4^{\prime}-6^{\prime}$-hump), while the full-band counts show an additional steep rise at small distances of $r<2^{\prime}$.

(3) The radial profiles of the background-subtracted excess counts confirm the statistical significance of both radial features. The centrally peaked point source excess within the average cluster radius $R_{200}$ is consistent with various previous studies, most of them conducted at lower redshifts. The detected (incomplete) average excess per cluster is found to be about one point source per system with evidence for lower X-ray luminosities below the soft-band detection limit.

(4) A second analysis was performed with all source lists rotated about the cluster center in a way that the principal elongation axes of the cluster emission are aligned for all systems. With the interpretation that the cluster elongation points in the direction of the main cluster assembly axis the tentative conclusion can be derived that the observed AGN activity is mostly occurring along these identified matter infall directions.

(5) The second outer radial feature at cluster-centric distances of 2-3 Mpc was previously reported also for 
an intermediate-redshift cluster sample by Ruderman and Ebeling [6]. However, a systematic boosting of this feature by foreground structures cannot be fully ruled out with the statistics of the present sample. The cross-correlation of public spectroscopic redshift information with the X-ray data, on the other hand, confirms the presence of a significant population of X-ray AGN in the cluster environments beyond a projected distance of $2 \mathrm{Mpc}$. The observed $4^{\prime}-$ $6^{\prime}$ hump feature is consistent with a population of bright soft-band-detected AGN triggered at a project distance of about $3 \times R_{200}$.

(6) Taking all results at face value lends support to the idea of two different AGN populations and triggering mechanisms of nuclear activity in the distant cluster environments. In this picture, the fiducial cluster regions inside $R_{200}$ of high- $z$ X-ray luminous systems harbor low-luminosity AGN triggered by close galaxy encounters in the infall regions, while the excess in the outer cluster environment at distances of 2-3 Mpc is due to major-merger induced AGN activity.

(7) With respect to distant cluster applications with the upcoming all-sky survey eROSITA, the results suggest that the spectroscopic temperature analysis of samples of distant cluster sources may be biased highly due to embedded point sources, while the detection and flux measurements in the soft band may not be significantly influenced in the general case.

The presented results are based on the currently largest homogeneously selected sample of X-ray luminous clusters at $z>0.9$. Statistical improvements and tests of the discussed results will require significantly larger, well-defined samples of distant X-ray clusters of about 50 objects, which should soon be available. The ultimate experiment of X-ray AGN in distant cluster environments at $r>1 \mathrm{Mpc}$ will be made possible by eROSITA in the near future.

\section{Acknowledgments}

The authors thank the anonymous referee for insightful comments that helped to improve the clarity of the paper. They thank the XDCP team members for carrying out the distant cluster survey. R. Fassbender would like to thank Gabriel Pratt for his comments, Andrea Merloni for helpful discussions, and Angela Bongiorno for the invitation to the special issue. This research was supported by the DFG cluster of excellence "Origin and Structure of the Universe" (http://www.universe-cluster.de/) and by the DFG under Grants BO 702/16-3. The XMM-Newton project is an ESA Science Mission with instruments and contributions directly funded by ESA Member States and the USA (NASA). This research has made use of the NASA/IPAC Extragalactic Database (NED) which is operated by the Jet Propulsion Laboratory, California Institute of Technology, under contract with the National Aeronautics and Space Administration.

\section{References}

[1] M. Branches, I. M. Gioia, C. Fanti, R. Fanti, and N. Cappelluti, "Chandra point-source counts in distant galaxy clusters," Astronomy and Astrophysics, vol. 462, no. 2, pp. 449-458, 2007.

[2] N. Cappelluti, M. Cappi, M. Dadina et al., "X-ray source overdensities in Chandra distant cluster fields: a new probe to map the cosmic tapestry?" Astronomy and Astrophysics, vol. 430, no. 1, pp. 39-45, 2005.

[3] J. Eastman, P. Martini, G. Sivakoff, D. D. Kelson, J. S. Mulchaey, and K. V. Tran, "First measurement of a rapid increase in the AGN fraction in high-redshift clusters of galaxies," Astrophysical Journal, vol. 664, no. 1, pp. L9-L12, 2007.

[4] R. Gilmour, P. Best, and O. Almaini, "The distribution of active galactic nuclei in a large sample of galaxy clusters," Monthly Notices of the Royal Astronomical Society, vol. 392, no. 4, pp. 1509-1531, 2009.

[5] P. Martini, G. R. Sivakoff, and J. S. Mulchaey, "The evolution of active galactic nuclei in clusters of galaxies to redshift 1.3," Astrophysical Journal Letters, vol. 701, no. 1, pp. 66-85, 2009.

[6] J. T. Ruderman and H. Ebeling, "The origin of the spatial distribution of X-ray-luminous active galactic nuclei in massive galaxy clusters," Astrophysical Journal, vol. 623, no. 2, pp. L81L84, 2005.

[7] E. Koulouridis and M. Plionis, "Luminous X-ray active galactic nuclei in clusters of galaxies," Astrophysical Journal Letters, vol. 714, no. 2, pp. L181-L184, 2010.

[8] O. Johnson, P. N. Best, and O. Almaini, "The content of active galactic nuclei in the $z=0.83$ cluster MS 1054-0321," Monthly Notices of the Royal Astronomical Society, vol. 343, no. 3, pp. 924-932, 2003.

[9] N. Rumbaugh, D. D. Kocevski, R. R. Gal et al., "The evolution and environments of X-ray emitting active galactic nuclei in high-redshift large-scale structures," The Astrophysical Journal, vol. 746, p. 155, 2012.

[10] M. Hilton, E. Lloyd-Davies, S. A. Stanford et al., "The Xmm cluster survey: active galactic nuclei and starburst galaxies in xmmxcs J2215.9-1738 at $z=1.46$," The Astrophysical Journal, vol. 718, no. 1, p. 133, 2010.

[11] M. Pierre, N. Clerc, B. Maughan et al., "A Chandra view of the $z=1.62$ galaxy cluster IRC-0218A," Astronomy \& Astrophysics, vol. 540, article A4, 2012.

[12] A. Galametz, D. Stern, P. R. M. Eisenhardt et al., "The cosmic evolution of active galactic nuclei in galaxy clusters," Astrophysical Journal Letters, vol. 694, no. 2, pp. 1309-1316, 2009.

[13] P. R. M. Eisenhardt, M. Brodwin, A. H. Gonzalez et al., "Clusters of galaxies in the first half of the universe from the IRAC shallow survey," Astrophysical Journal Letters, vol. 684, no. 2, pp. 905-932, 2008.

[14] R. Fassbender, H. Bohringer, A. Nastasi et al., "The x-ray luminous galaxy cluster population at $0.9<z \lesssim 1.6$ as revealed by the XMM-Newton distant cluster project," New Journal of Physics, vol. 13, Article ID 125014, 2011.

[15] R. Fassbender, Studying cosmic evolution with the XMMNewton distant cluster project: $x$-ray luminous galaxy clusters at $z \sim>1$ and their galaxy populations, Ph.D. thesis, Ludwig Maximilians University Munich, 2007, astro-ph/0806.0861.

[16] H. Bohringer, C. R. Mullis, P. Rosati et al., "Galaxy cluster archaeology," in Proceedings of the ESO Messenger, vol. 120, pp. 33-36, 2005. 
[17] P. Predehl, R. Andritschke, H. Bohringer et al., "eROSITA on SRG," in Proceedings of the Society of PhotoOptical Instrumentation Engineers SPIE Conference Series, vol. 7732, Society of Photo-Optical Instrumentation Engineers (SPIE), 2010.

[18] R. Fassbender, H. Böhringer, J. S. Santos et al., "A panchromatic view of the galaxy cluster XMMU J1230.3 + 1339 at $z=0.975$ : observing the assembly of a massive system," Astronomy and Astrophysics, vol. 527, no. 10, article A78, 2011.

[19] N. Cappelluti, G. Hasinger, M. Brusa et al., "The XMMNewton wide-field survey in the COSMOS field. II. X-ray data and the $\log$ N-log S relations," Astrophysical Journal, vol. 172, no. 1, pp. 341-352, 2007.

[20] R. Gobat, E. Daddi, M. Onodera et al., "A mature cluster with X-ray emission at $z=2.07$," Astronomy and Astrophysics, vol. 526, no. 16, article A133, 2011.

[21] P. Rosati, P. Tozzi, R. Gobat et al., "Multi-wavelength study of XMMU J2235.3 - 2557: the most massive galaxy cluster at $z>1$," Astronomy \& Astrophysics, vol. 508, no. 2, pp. 583-591, 2009.

[22] P. Rosati, P. Tozzi, S. Ettori et al., "Chandra and XMM-Newton observations of RDCS 1252.9 - 2927, a massive cluster at $z=1.241$," Astronomical Journal, vol. 127, no. 1, pp. 230-238, 2004.

[23] B. J. Maughan, L. R. Jones, M. Pierre et al., "Testing the galaxy cluster mass-observable relations at $z=1$ with XMM-Newton and Chandra observations of XLSSJ022403.9041328," Monthly Notices of the Royal Astronomical Society, vol. 387, no. 3, pp. 998-1006, 2008.

[24] S. Giodini, D. Pierini, A. Finoguenov et al., "Stellar and total baryon mass fractions in groups and clusters since redshift," Astrophysical Journal Letters, vol. 703, no. 1, pp. 982-993, 2009.

[25] D. J. Croton, V. Springel, S. D. M. White et al., "The many lives of active galactic nuclei: cooling flows, black holes and the luminosities and colours of galaxies," Monthly Notices of the Royal Astronomical Society, vol. 365, no. 1, pp. 11-28, 2006.

[26] J. S. Santos, R. Fassbender, A. Nastasi et al., "Discovery of a massive X-ray luminous galaxy cluster at $z=1.579$," Astronomy and Astrophysics, vol. 531, article L15, 2011.

[27] R. Fassbender, A. Nastasi, H. Bohringer et al., "The X-ray luminous galaxy cluster XMMU J1007.4 + 1237 at $z=$ 1.56. The dawn of starburst activity in cluster cores," Astronomy and Astrophysics, vol. 527, article L10, 2011.

[28] A. Nastasi, R. Fassbender, H. Böhringer et al., "Discovery of the X-ray selected galaxy cluster XMMU J0338.8+0021 at $z=$ 1.49: indications of a young system with a brightest galaxy in formation," Astronomy and Astrophysics, vol. 532, article L6, 2011.

[29] S. A. Stanford, A. K. Romer, K. Sabirli et al., "The XMM cluster survey: a massive galaxy cluster at $z=1.45$," Astrophysical Journal, vol. 646, no. II, pp. L13-L16, 2006.

[30] M. Hilton, C. A. Collins, S. A. Stanford et al., "The XMM cluster survey: the dynamical state of XMMXCS J2215.9-1738 at $z=1.457$," Astrophysical Journal Letters, vol. 670, no. 2, pp. 1000-1009, 2007.

[31] M. Hilton, S. A. Stanford, J. P. Stott et al., “The xmm cluster survey: galaxy morphologies and the color-magnitude relation in XMMXCS J2215.9 - 1738 at $z=1.46$," Astrophysical Journal Letters, vol. 697, no. 1, pp. 436-451, 2009.

[32] C. R. Mullis, P. Rosati, G. Lamer et al., "Discovery of an X-rayluminous galaxy cluster at $z=1.4$," Astrophysical Journal, vol. 623, no. 2, pp. L85-L88, 2005.
[33] R. Šuhada, R. Fassbender, A. Nastasi et al., "Exploring the galaxy cluster-group transition regime at high redshifts: physical properties of two newly detected $z>1$ systems," Astronomy and Astrophysics, vol. 530, article A110, 2011.

[34] G. Wilson, A. Muzzin, H. K. C. Yee et al., "Spectroscopic confirmation of a massive red-sequence-selected galaxy cluster at $z=1.34$ in the sparcs-south cluster Survey," The Astrophysical Journal, vol. 698, p. 1943, 2009.

[35] A. de Hoon et al., submitted to. Astronomyand Astrophysics.

[36] K. S. Dawson, G. Aldering, R. Amanullah et al., "An intensive hubble space telescope survey for $z>1$ type Ia supernovae by targeting galaxy clusters," Astronomical Journal, vol. 138, no. 5, pp. 1271-1283, 2009.

[37] D. Pierini, R. Suhada, R. Fassbender et al., "First simultaneous optical/near-infrared imaging of an X-ray selected, high-redshift cluster of galaxies with GROND: the galaxy population of XMMU J0338.7 + 0030 at $z=1.1$," Astronomy \& Astrophysics, vol. 540, article A45, 2012.

[38] A. D. Schwope, G. Lamer, A. de Hoon et al., "XMMU J100750.5 + 125818: a strong lensing cluster at $z=1.082$," Astronomy and Astrophysics, vol. 513, no. 11, article L10, 2010.

[39] F. Pacaud, M. Pierre, C. Adami et al., "The XMM-LSS survey: the Class 1 cluster sample over the initial 5 deg 2 and its cosmological modelling," Monthly Notices of the Royal Astronomical Society, vol. 382, no. 3, pp. 1289-1308, 2007.

[40] C. Adami, A. Mazure, M. Pierre et al., "The XMM-LSS survey: optical assessment and properties of different X-ray selected cluster classes," Astronomy and Astrophysics, vol. 526, no. 2, article A18, 2010.

[41] J. S. Santos, P. Rosati, R. Gobat et al., "Multiwavelength observations of a rich galaxy cluster at $\mathrm{z} \sim 1$ : the HST/ACS colourmagnitude diagram," Astronomy and Astrophysics, vol. 501, no. 1, pp. 49-60, 2009.

[42] R. Fassbender, H. Böhringer, G. Lamer et al., "Indications for $3 \mathrm{Mpc}$-scale large-scale structure associated with an Xray luminous cluster of galaxies at $z=0.95$," Astronomy and Astrophysics, vol. 481, no. 2, pp. L73-L77, 2008.

[43] D. P. Schneider, P. B. Hall, G. T. Richards et al., "The sloan digital sky survey quasar catalog. IV. Fifth data release," Astronomical Journal, vol. 134, no. 1, pp. 102-117, 2007.

[44] F. J. Carrera, J. Ebrero, S. Mateos et al., "The XMM-Newton serendipitous survey III. the AXIS X-ray source counts and angular clustering," Astronomy and Astrophysics, vol. 469, no. 1, pp. 27-46, 2007.

[45] X. Barcons, F. J. Carrera, M. T. Ceballos et al., "The XMMNewton serendipitous survey," Astronomy and Astrophysics, vol. 476, no. 3, pp. 1191-1203, 2007.

[46] C. Feruglio, F. Fiore, F. La Franca et al., "The XMM-Newton survey of the ELAIS-S1 field - II. Optical identifications and multiwavelength catalogue of X-ray sources," Astronomy and Astrophysics, vol. 488, no. 1, pp. 417-428, 2008.

[47] N. Sacchi, F. La Franca, C. Feruglio et al., "Spectroscopic identifications of spitzer sources in the swire/xmm-newton/elaiss1 field: a large fraction of active galactic nuclei with high $\mathrm{f}(24$ $\mu \mathrm{m}) / \mathrm{f}(\mathrm{r})$ ratio," Astrophysical Journal Letters, vol. 703, no. 2, pp. 1778-1790, 2009.

[48] M. Tanaka, C. Lidman, R. G. Bower et al., "Star formation activities of galaxies in the large-scale structures at $z=1.2$," Astronomy and Astrophysics, vol. 507, no. 2, pp. 671-682, 2009.

[49] M.-P. Veron-Cetty and P. Veron, "A catalogue of quasars and active nuclei: 10th edition," Astronomy and Astrophysics, vol. 374, pp. 92-94, 2001.

[50] O. Le Fèvre, G. Vettolani, B. Garilli et al., "The VIMOS VLT deep survey: first epoch VVDS-deep survey: 11564 spectra 
with $17.5 \leq \mathrm{iAB} \leq 24$, and the redshift distribution over $0 \leq z \leq 5$," Astronomy and Astrophysics, vol. 439, no. 3, pp. 845-862, 2005.

[51] X. Barcons, F. J. Carrera, M. G. Watson et al., "The XMMNewton serendipitous survey: II. First results from the AXIS high galactic latitude medium sensitivity survey," Astronomy and Astrophysics, vol. 382, no. 2, pp. 522-536, 2002. 

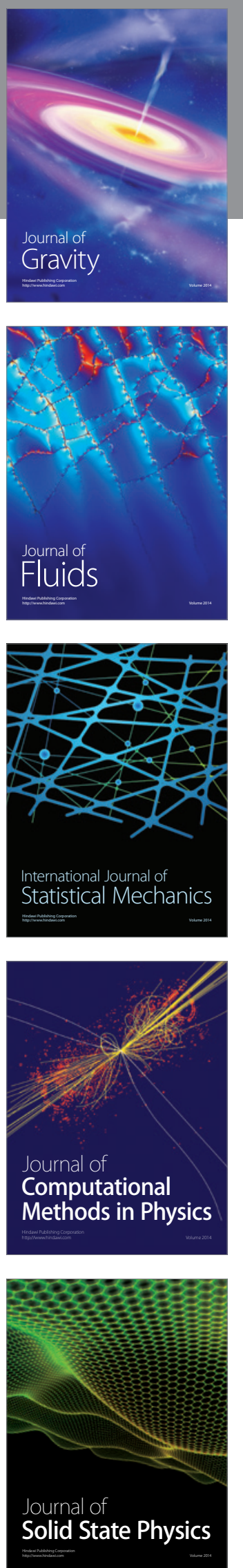

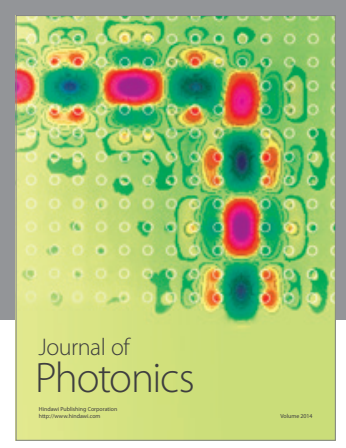

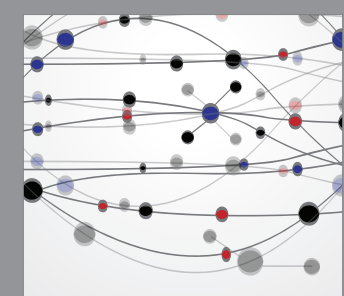

The Scientific World Journal
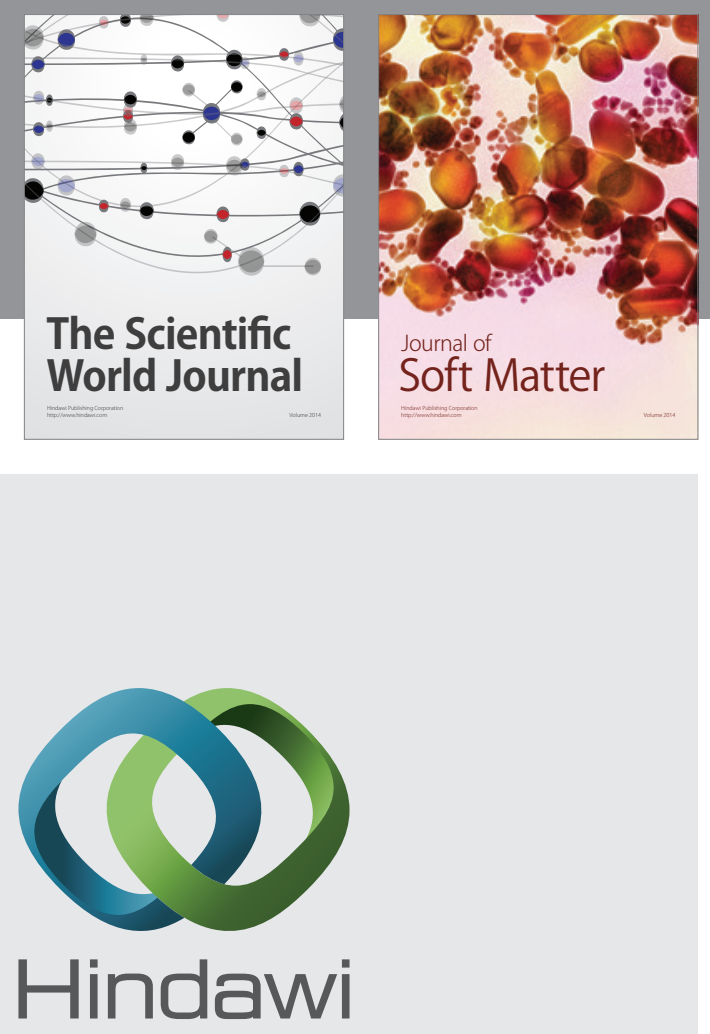

Submit your manuscripts at

http://www.hindawi.com
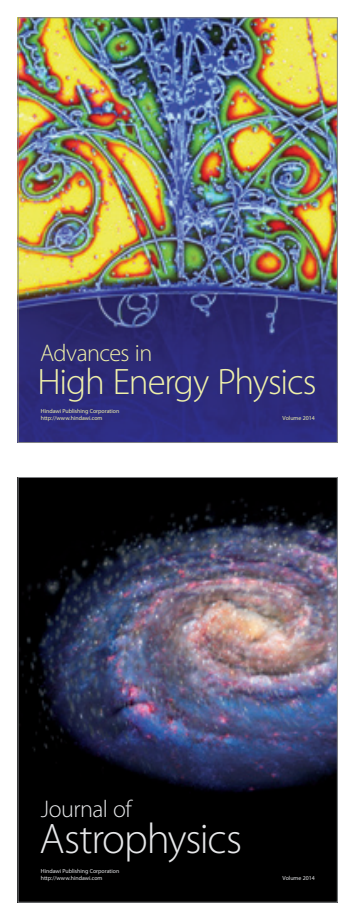
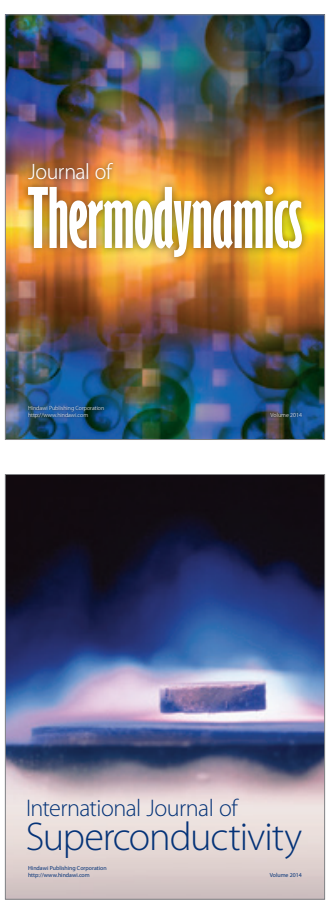
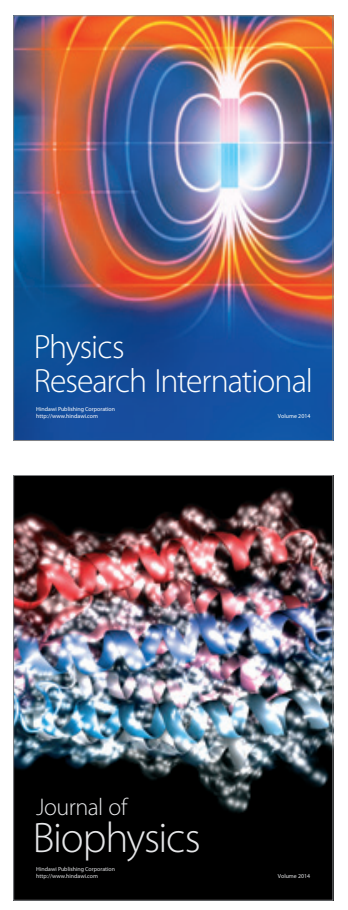
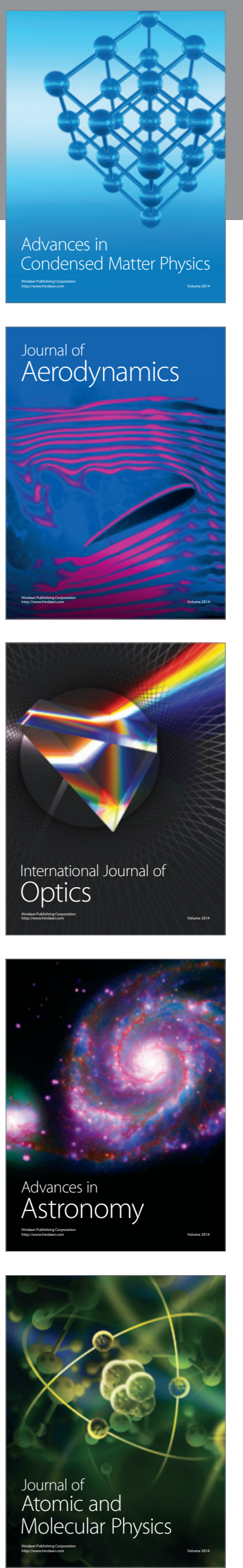